\title{
Analytic Evaluation of Target Detection in Heterogeneous Wireless Sensor Networks ${ }^{\dagger}$
}

\author{
LOUKAS LAZOS \\ University of Arizona \\ RADHA POOVENDRAN and JAMES A. RITCEY \\ University of Washington
}

\begin{abstract}
In this article, we address the problem of target detection in Wireless Sensor Networks (WSN). We formulate the target detection problem as a line-set intersection problem and use integral geometry to analytically characterize the probability of target detection for both stochastic and deterministic deployments. Compared to previous work, we analyze WSNs where sensors have heterogeneous sensing capabilities. For the stochastic case, we evaluate the probability that the target is detected by at least $k$ sensors and compute the free path until the target is first detected. For the deterministic case, we show an analogy between the target detection problem and the problem of minimizing the average symbol error probability in 2-dimensional digital modulation schemes. Motivated by this analogy, we propose a heuristic sensor placement algorithm called DATE, that makes use of well known signal constellations for determining good WSN constellations. We also propose a heuristic called CDATE for connected WSN constellations, that yields high target detection probability.
\end{abstract}

Categories and Subject Descriptors: C.2 [Computer System Organization]: Computer - Communication Networks; C.2.1 [Network Architecture and Design]: Distributed NetworksNetwork Topology

General Terms: Algorithm, Design, Performance

Additional Key Words and Phrases: Target Detection, Wireless Sensor Networks, Heterogeneous

\section{INTRODUCTION}

One of the fundamental services provided by Wireless Sensor Networks (WSN) is the surveillance of a Field of Interest $(F o I)$. A number of wireless sensors may

${ }^{\dagger}$ This work was supported in part by the following grants: ONR YIP award, N00014-04-1-0479, ARO PECASE grant, W911NF-05-1-0491, ARL CTA Grant DAAD 19-01-2-0011, and ARO MURI Grant \#W 911 NF 0710287. This document was prepared through collaborative participation in the Communications and Networks Consortium sponsored by the US Army Research Laboratory under the Collaborative Technology Alliance Program, DAAD19-01-2-0011. The US Government is authorized to reproduce and distribute reprints for Government purposes notwithstanding any copyright notation thereon. The views and conclusions contained in this document are those of the author and should not be interpreted as representing the official policies, either expressed or implied, of the Army Research Laboratory or the US Government. Preliminary versions of this article have appeared in WiOpt 2007 [Lazos et al. 2007a], and IPSN 2007 [Lazos et al. 2007b]. Permission to make digital/hard copy of all or part of this material without fee for personal or classroom use provided that the copies are not made or distributed for profit or commercial advantage, the ACM copyright/server notice, the title of the publication, and its date appear, and notice is given that copying is by permission of the ACM, Inc. To copy otherwise, to republish, to post on servers, or to redistribute to lists requires prior specific permission and/or a fee.

(C) $20 \mathrm{YY}$ ACM 0000-0000/20YY/0000-0001 $\$ 5.00$ 
be deployed within an FoI to monitor any ongoing activity. As an example, in a military scenario, WSNs may be used to detect intrusion into restricted areas. In a habitat monitoring environment, a WSN may be used to detect the motion of animals within part of the forest or close to a specific area such as a river. Target detection can be realized via one or multiple sensing modalities such as optical, mechanical, acoustic, thermal, RF and magnetic sensing. In fact, to ensure robustness and enhance performance, oftentimes a sensor fusion approach is required [Koushanfar et al. 2002]. In such a case, target detection can be realized via fusion of data aggregated from modalities such as infrared, CCD, pressure and acoustic.

Depending on the modality, sensing areas can have any arbitrary shape, a reality significantly different from the widely adopted idealized unit disk model [Gui and Mohapatra 2004; Cao et al. 2005; Aslam et al. 2003; Cho 2002; Yang and Sikdar 2003; Dousse et al. 2006; Kumar et al. 2005; Meguerdichian et al. 2001; Clouqueur et al. 2002; Clouqueur et al. 2004]. A CCD camera, for example, can have a conic sensing area. Moreover, in sensor fusion scenarios, devices of different modalities have heterogeneous sensing capabilities. Furthermore, even if devices with identical sensing capabilities are deployed, the morphology of the FoI (physical obstacles, variation in physical conditions such as temperature, humidity, sound levels), can yield a highly heterogeneous network.

For the purposes of target detection, the sensor deployment can be either stochastic or deterministic depending on the application and the FoI. Stochastic deployment is preferred when the $F O I$ is not under the designer's control at the time of deployment, or it is more cost-effective to randomly deploy the sensors (large-scale networks) [Cao et al. 2005; Meguerdichian et al. 2001; Clouqueur et al. 2004; Lazos et al. 2007b]. On the other hand, when a limited number of sensors are available for monitoring the FoI, deterministic deployment avoids WSN constellations with poor performance. In this article, we address the problem of analytically evaluating the probability of target detection in heterogeneous sensor networks, under both stochastic and deterministic deployment.

\subsection{Our Contributions}

We map the target detection problem to a line-set intersection problem. Based on our mapping, we use tools from integral geometry to analytically evaluate the probability of detecting targets crossing an FoI, for both stochastic and deterministic deployments. Our mapping allows us to consider a heterogeneous sensing model where sensors need not have identical sensing capabilities.

For the stochastic network deployment case, we analytically evaluate the probability of detecting a target $X$, when $X$ crosses the $F o I$ moving on a random line trajectory, and $N$ sensors are randomly deployed. Our results show that the probability of target detection is independent of the shape of the sensing areas, but only depends on the length of the perimeters of the sensing areas ${ }^{1}$.

For the deterministic sensor deployment case, we show that the complexity of the analytic formula of target detection probability grows exponentially with the network size and, hence, is not practical for large networks. We therefore, derive relevant lower and upper bounds. We further show that the problem of finding the

\footnotetext{
${ }^{1}$ Or the length of the perimeter of the convex hull of the sensing areas, for non-convex shapes.

ACM Journal Name, Vol. V, No. N, Month 20YY.
} 
WSN constellation that maximizes the lower bound, is analogous to the problem of finding the signal constellation that minimizes the average symbol error probability in digital modulation schemes over Additive White Gaussian Noise (AWGN) channels. The latter problem can be addressed by solving the circle packing problem. Utilizing well known signal constellations, we propose a heuristic sensor placement algorithm called DATE which stands for Deterministic plAcement for Target dEtection. We also propose a heuristic placement algorithm called CDATE (Connected DATE) to generate $k$-connected WSNs with high target detection capability.

\subsection{Paper Organization}

The rest of the paper is organized as follows. In Section 2, we present related work. In Section 3, we state our model assumptions and formulate the target detection problem. In Section 4, we analytically evaluate the probability of target detection for random deployment of $N$ sensors and in Section 5, we consider the deterministic sensor deployment case. In Section 6, we present the analogy of the target detection problem to the 2-dimensional digital modulation scheme problem and propose a heuristic placement algorithm called DATE. In Section 7, we present CDATE, a heuristic placement algorithm that yields connected networks. In Section 8, we present our performance evaluation and in Section 9, we present our conclusions.

\section{RELATED WORK}

The target detection problem in WSN has been a topic of extensive study under different metrics and assumptions [Gui and Mohapatra 2004; Cao et al. 2005; Aslam et al. 2003; Cho 2002; Yang and Sikdar 2003; Dousse et al. 2006; Kumar et al. 2005; Meguerdichian et al. 2001; Clouqueur et al. 2002; Clouqueur et al. 2004; Lazos et al. 2007a; Lazos et al. 2007b].

Gui and Mohapatra 2004, investigate the tradeoff between detection quality and power conservation. They assume that nodes are randomly deployed within a planar FoI, and have sensing areas that follow the unit disk model. Given a target $X$ moving on a straight line, they derive the mean time until $X$ is first detected. They also provide sleeping scheduling algorithms that lead to power conservation, while guaranteeing a minimum response time for detecting a target crossing the FoI.

Cao et al. 2005, provide analytic formulas for the mean delay until a target is detected, when targets move on a straight line at a constant speed. The authors consider random deployment of $N$ sensors with each sensor having identical sensing areas that follow the unit disk model. They provide closed analytic formulas on target detection probability that take into account the sleeping pattern of sensors.

Meguerdichian et al. 2001, propose a collaborative detection model, where sensors collectively arrive at a consensus about the presence of a target. The problem addressed in [Meguerdichian et al. 2001] is coverage of the FoI, but their formulation can be indirectly used to also evaluate the target detection probability. They assume that the detection capability of each sensor decays as a function of distance and hence, the sensing area of each sensor follows the unit disk model.

Clouqueur et al. 2004, adopt the model in [Meguerdichian et al. 2001], with sensors being randomly deployed within the FoI. The authors formulate the target detection problem as an unauthorized traversal problem and propose deployment strategies for minimizing the cost of the network that achieves the desired target 
detection probability. They also propose a deployment strategy where only part of the available sensors are randomly deployed. If the partial deployment satisfies the performance metric, no more sensors are deployed. Otherwise the process is repeated until the performance threshold is met.

Kumar et al. 2005, address the problem of optimum $k$-coverage of the boundary of an FoI. Covering the boundary of an $F o I$ guarantees that any intruder will be detected with certainty. The authors assume that all sensors have identical sensing areas following the unit disk model as well. While target detection is guaranteed when the boundary of the $F o I$ is covered, when the number of sensors available are not sufficient to cover the boundary, placement at the perimeter of the $F o I$ does not yield the maximum target detection probability.

Dousse et al. 2006 address the problem of determining the delay until a target (intruder) is first detected. They consider the detection problem under the additional constraint that any sensor detecting the target must have a connected path to the sink. They assume that targets move in a straight line, and all sensors have identical sensing areas conforming to the unit disk model.

\section{MODEL ASSUMPTIONS, PROBLEM FORMULATION AND BACKGROUND}

\subsection{Notation}

$N$ : Number of sensors for deployment.

$\mathcal{A}_{0}$ : $\quad$ Field of Interest, with perimeter $L_{0}$, and area $F_{0}$.

$\mathcal{A}_{i}$ : Sensing area of node $s_{i}, i=1 \ldots N$, with perimeter $L_{i}$, and area $F_{i}$.

$\ell(\xi, \theta)$ : Line $\ell$ with distance $\xi$, from the origin and angle $\theta$.

$d_{i, j}: \quad$ Pairwise distance among sensors $s_{i}, s_{j}$.

$S_{i}$ : A bounded and connected set of points in the plane with perimeter $L_{i}$.

$X$ : Target crossing the FoI.

$P_{D}: \quad$ Probability of target detection by at least one sensor.

$P_{D}(k)$ : Probability that a target $X$ is detected by at least $k$ sensors.

$Z_{N, k}: \quad$ All $\left(\begin{array}{c}N \\ k\end{array}\right) k$-tuples of a vector $[1, \ldots, N]$.

$P\left(Z_{N, k}\right)$ : Probability that a target $X$ is detected by exactly $k$ sensors.

$m(\ell)$ : Measure of a set of lines.

$T_{i}(\theta)$ : The thickness of a set $S_{i}$ at direction $\theta$.

$E(T)$ : Average thickness of a set $S$.

\subsection{Model Assumptions}

Sensor Deployment: We assume that the WSN consists of $N$ sensors, that can, (a) be identically, and independently distributed within the FoI, according to the random distribution and, (b) be placed at any desired position within the FoI in a deterministic way. The $F_{O} I$ is assumed to be a connected and closed set of perimeter $L_{0}$ and area $F_{0}$ and of arbitrary shape. In the case where the $F_{o} I$ is not convex, the perimeter $L_{0}^{h}$, of the convex hull of $F o I$ is assumed to be known.

Target Model: The target $X$ is assumed to move on a straight line trajectory, with all trajectories crossing the FoI being equiprobable. Considering equiprobable target trajectories is a reasonable assumption, when the target (intruder) has no knowledge of the sensor placement so as to bias his trajectory within the FoI. Furthermore, many semantics used by sensors to detect a target such as electro- 


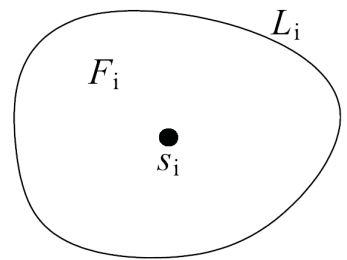

(a)

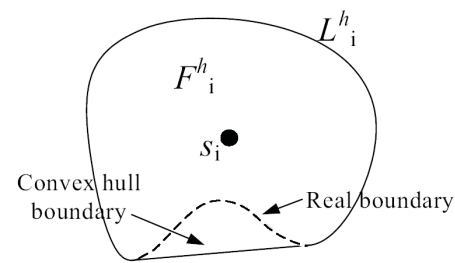

(b)

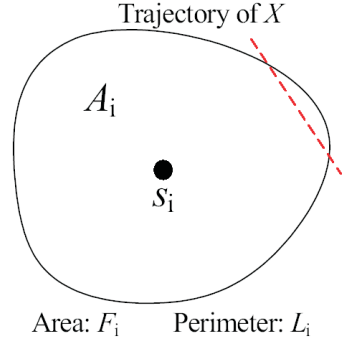

(c)

Fig. 1. (a) A convex sensing area $\mathcal{A}_{i}$ of size $F_{i}$ and perimeter $L_{i}$, (b) a non-convex sensing area with a convex hull boundary of size $L_{i}^{h}$ and area size $F_{i}^{h}$, (c) the instant detection model: a target $X$ is detected if the trajectory of $X$ crosses the sensing area of $s_{i}$.

magnetic or acoustic signals propagate in straight lines.

In addition, straight line trajectories yield a worst case analysis for the probability of detection. Given any arbitrary entry and exit point in the FoI, moving on a straight line minimizes the length of the trajectory of the target within the $F_{o I}$ (therefore minimizing the time that the target can be detected). The worst case analysis allows us to compute network parameters such as sensor density and length of the perimeters of the sensing areas, so that minimum guarantees on target detection can be provided. Finally, straight line motion models have also been assumed in previous works addressing the target detection problem [Gui and Mohapatra 2004; Cao et al. 2005; Dousse et al. 2006].

Sensing Model: We assume that each sensor $s_{i}, i=1 \ldots N$ has a sensing area $A_{i}$ that is a closed and connected set of perimeter $L_{i}$ and area $F_{i}$. In the case of non-convex shapes, we assume that the perimeter, denoted as $L_{i}^{h}$, of the convex hull of $A_{i}$ is known. Based on our assumptions, sensors need not have an identical sensing area $A_{i}$. For detecting a target $X$, we assume the Boolean detection model, where a target $X$ is detected by a sensor $s_{i}$ if the trajectory of $X$ crosses the sensing area of $s_{i}$. The Boolean detection model is shown in figure 1(c), and has also been assumed in [Cao et al. 2005; Kumar et al. 2005; Kumar et al. 2005]. Figure 1(a) illustrates a sensing area $\mathcal{A}_{i}$ of convex shape. Figure 1(b) illustrates a non-convex sensing area and the equivalent convex hull boundary.

\subsection{Problem Formulation}

The mobile target detection problem can be formulated as follows.

Mobile target detection problem: Given an FoI $\mathcal{A}_{0}$ of perimeter $L_{0}$ and $N$ sensors with sensor $s_{i}$ having a sensing area $\mathcal{A}_{i}$ of perimeter $L_{i}$, and a target $X$ crossing the FoI while moving on a straight line trajectory compute, (a) the probability $P_{D}$ of detecting $X$ when $N$ sensors are randomly deployed within the FoI and, (b) the deterministic WSN constellation that maximizes $P_{D}$.

Mapping the mobile target detection problem: The mobile target detection problem can be mapped to the following line-set intersection problem. Let the $F_{o I}$ be mapped to a bounded set $S_{0}$, defined as a collection of points in the plane with perimeter length $L_{0}$. Let the sensing area of sensor $s_{i}$ be mapped to a bounded 
set $S_{i}$ with perimeter length $L_{i}$. Let the trajectory of the target $X$ be mapped to a straight line $\ell(\xi, \theta)$ in the plane, with parameters $\xi$ and $\theta$ being the shortest distance of $\ell$ to the origin of a coordinate system, and $\theta$ being the angle of the line perpendicular to $\ell$ with respect to the $x$ axis. Then, the mobile target detection problem for WSN is equivalent to the following line-set intersection problem.

Line-set intersection problem: Given a bounded set $S_{0}$ of perimeter length $L_{0}$, $N$ sets $S_{i}$ of perimeter length $L_{i}$, and a random line $\ell(\xi, \theta)$ intersecting $S_{0}$ compute, (a) the probability $P_{D}$ that a random line also intersects with any of the $N$ sets $S_{i}$, when the sets are randomly deployed and, (b) the deterministic set constellation that maximizes $P_{D}$.

The line-set intersection problem can be addressed with tools from Integral Geometry [Santaló 2004; Solomon 1978]. A brief background on Integral Geometry is presented in Appendix 1.

\section{TARGET DETECTION UNDER RANDOM SENSOR DEPLOYMENT}

In this section, we analytically evaluate the detection probability $P_{D}(k)$ of a target $X$ crossing the $F_{O} I$ by at least $k$ sensors, for the case of random sensor deployment. We then compute the mean time until $X$ is first detected. The $P_{D}(k)$ is given by the following theorem.

TheOREM 1. Let $\mathcal{A}_{0}$ be a bounded FoI of perimeter length $L_{0}$ monitored by $N$ sensors randomly deployed within $\mathcal{A}_{0}$, with sensor $s_{i}, i=1 \ldots N$ having a sensing area of perimeter length $L_{i}$. The probability $P_{D}(k)$ that at least $k \geq 1$ sensors detect a target $X$ crossing the FoI is given by:

$$
P_{D}(k)=1-\sum_{w=0}^{k-1} \sum_{j=1}^{\left|Z_{N, w}\right|} \prod_{i=1}^{\left|z_{j}\right|} q_{z_{j}(i)} \prod_{v=1}^{|\bar{z} j|}\left(1-q_{\bar{z}_{j}(v)}\right),
$$

where $Z_{N, w}$ denotes the $\left(\begin{array}{c}N \\ w\end{array}\right)$ w-tuples $z_{j}$ of vector $[1, \ldots, N]$. That is, $Z_{N, w}=\left\{z_{j}\right.$ : $\left.z_{j(1)}, \ldots, z_{j(i)}, \ldots, z_{j(w)} \mid j(i) \in[1, N], j(i) \neq j(g), \forall i \neq g\right\}$. The $\bar{z}_{j}$ denotes the complement $(N-w)$-tuple of $z_{j}$ with respect to vector $[1, \ldots, N]$, and $q_{i}$ is given by $q_{i}=\frac{L_{i}}{L_{0}}$.

Proof. The proof is provided in Appendix 2.

From Theorem 1, note that $P_{D}(k)$ depends only on the ratios $\frac{L_{i}}{L_{0}}$ of $\mathcal{A}_{i}$ and not the shape of the sensing areas. Hence, Theorem 1 allows the analytic computation of the detection probability in the case of sensors with heterogeneous sensing areas. If sensors have sensing areas with perimeters of equal length (not necessarily identical shapes), (1) can be simplified to the following form.

COROLlary 1 . The probability $P_{D}(k)$ when all sensors have sensing areas with equal perimeters $L$ is equal to:

$$
P_{D}(k)=1-\sum_{i=0}^{k-1}\left(\begin{array}{c}
N \\
i
\end{array}\right) \frac{L^{i}\left(L_{0}-L\right)^{N-i}}{L_{0}^{N}} .
$$

Proof. Corollary 1 follows by setting $q_{i}=\frac{L}{L_{0}}$ in (1). 
Using Theorem 1, we can also evaluate the probability that a target $X$ remains undetected. Corollary 2, computes the probability $P_{M}$ of missing a target.

Corollary 2. The probability $P_{M}$ of missing a target $X$ is equal to:

$$
P_{M}=\prod_{i=1}^{N}\left(1-\frac{L_{i}}{L_{0}}\right) .
$$

Proof. The proof of Corollary 2 follows, by observing that $P_{M}=P\left(Z_{N, 0}\right)$, and $z_{j}=\emptyset, \bar{z}_{j}=\{1, \ldots, N\}$. The $P\left(Z_{N, 0}\right)$ denotes the probability that a random line does not intersect any of the $N$ sensing areas.

Depending on the application, (3) allows us to select $L_{i}, N$ so that $P_{M}$ remains below any desired threshold value. Theorem 1, can also be used to describe the target detection capability of a WSN, at a particular direction $\theta$. The measure of a set of lines intersecting with a set $S$ at a fixed direction $\theta$ is equal to the thickness $T(\theta)$ of the set in that direction. In brief, the thickness $T(\theta)$ of a set $S$ at a direction $\theta$ is a measure of the set of lines crossing $S$ at a direction perpendicular to $\theta .^{2}$ Hence, the probability $q_{i}(\theta)$ that a target $X$ is detected by a single sensor when $X$ is moving at direction $\theta$ is:

$$
q_{i}(\theta)=\frac{T_{i}(\theta)}{T_{0}(\theta)} .
$$

Substituting (4) to (1), yields the probability $P_{D}(k, \theta)$ at a particular direction $\theta$. The probability $P_{D}(k, \theta)$, can be used to evaluate the target detection capability of a WSN, when the possible target trajectories follow a distribution $f(\theta)$. In such a case, the target detection probability $P_{D}(k)$ is expressed in the following corollary.

COROLlary 3 . The probability $P_{D}(k)$ that at least $k \geq 1$ sensors detect a target $X$ crossing the FoI and moving on a straight line trajectory, with the line trajectories being distributed according to $f(\theta)$, is given by:

$$
P_{D}(k)=\int_{0}^{\pi} f(\theta)\left(1-\sum_{w=0}^{k-1} \sum_{j=1}^{\left|Z_{N, w}\right|\left|z_{j}\right|} \prod_{i=1}^{|\bar{z} j|} q_{z_{j}(i)}(\theta) \prod_{v=1}^{z}\left(1-q_{\bar{z}_{j}(v)}(\theta)\right)\right) d \theta
$$

where $f(\theta)$ denotes the target trajectory distribution with respect to the direction $\theta, Z_{N, w}$ denotes the $\left(\begin{array}{c}N \\ w\end{array}\right)$ w-tuples $z_{j}$ of vector $[1, \ldots, N]$. That is, $Z_{N, w}=\left\{z_{j}\right.$ : $\left.z_{j(1)}, \ldots, z_{j(i)}, \ldots, z_{j(w)} \mid j(i) \in[1, N], j(i) \neq j(g), \forall i \neq g\right\}$. The $\bar{z}_{j}$ denotes the complement $(N-w)$-tuple of $z_{j}$ with respect to vector $[1, \ldots, N]$, and $q_{i}(\theta)$ is given by $q_{i}(\theta)=\frac{T_{i}(\theta)}{T_{0}(\theta)}$.

Proof. For a given direction $\theta$ the target detection probability $P_{D}(k, \theta)$ is given by (1), by substituting $q_{i}$ with $q_{i}(\theta)=\frac{T_{i}(\theta)}{T_{0}(\theta)}$. Corollary 3 , follows by computing the average value of $P_{D}(k, \theta)$, over all $\theta$.

$$
P_{D}(k)=\int_{0}^{\pi} f(\theta) P_{D}(k, \theta) d \theta .
$$

\footnotetext{
${ }^{2}$ See Appendix 1 for a formal definition of the set thickness $T(\theta)$.
} 
The complexity of computing (1) and (5) grows exponentially with the heterogeneity of the sensing areas. If all sensors had sensing areas of different perimeters, an exponential number of terms must be summed to calculate (1),(5). For largescale networks, $P_{D}(k)$ can be approximated with the use of the following theorem.

TheOREM 2. Let the probability $q_{i}$ that a target $X$ is detected by sensor $s_{i}$ be small, while the sum of the probabilities $\sum_{i} q_{i}$ is nearly a constant $\gamma$ as $N \rightarrow \infty$. The probability $P\left(Z_{N, k}\right)$, that exactly $k$ sensors detect the target $X$, converges to a Poisson distribution of rate $\gamma$,

$$
P\left(Z_{N, k}\right)=\frac{\gamma^{k}}{k !} e^{-\gamma}, \quad \sum_{i} q_{i} \rightarrow \gamma, \quad \max _{i} q_{i} \rightarrow 0 .
$$

Proof. Theorem 2 is an application of the Poisson's law of rare events [Itô 1984].

Theorem 2 states that as the number of sensors deployed becomes large, the probability $P\left(Z_{N, k}\right)$ that a target $X$ is detected by exactly $k$ sensors, or equivalently, the probability that a random line intersects exactly $k$ sets, is Poisson distributed regardless of the heterogeneity of the sensing areas if: (a) the probabilities $q_{i}$ for any individual sensor to detect $X$ become infinitely small, a condition that holds if $L_{i}<<L_{0}, \forall i$ and, (b) the summation of all individual probabilities $q_{i}$ remains constant for an increasing $N$. The two conditions (a), (b) are satisfied when the area where the sensors are deployed grows with the increase of $N$ so that each individual probability $q_{i}$ is driven to zero, while the sensor deployment density remains constant. Hence, Theorem 2 is useful in the case of large networks covering an $F o I$ of large perimeter.

\subsection{Free Path Until the First Target Detection}

In many target detection applications, we are not only interested in the number of sensors able to detect a target, but also in how fast detection occurs. For example, in an area intrusion detection system it is critical that any intruder is detected in a timely manner.

A relevant metric of the timeliness of the detection of a moving target is the distance that such a target can travel within the FoI without being detected. We refer to such distance as the free path and denote its length by $\sigma$. In this section, we analytically evaluate the length of the free path $\sigma$. To facilitate our computations, we restrict our analysis to homogeneous sensing areas with constant thickness in all directions ${ }^{3}$. Examples of sensing area shapes with constant thickness in all directions the Reuleaux polygons [Reuleaux 1963] and the unit disk.

Theorem 3. Let $N$ sensors be deployed within the FoI of area $F_{0}$, with each sensor $s_{i}$ having a constant thickness $T_{i}(\theta)=E(T), \forall i$ in all possible directions $\theta$, where $E(T)$ denotes the average thickness of each set. The probability that the free path $\sigma$ before the target $X$ is detected is longer than a threshold value $\sigma_{x}$, is given by:

$$
P\left(\sigma \geq \sigma_{x}\right) \approx e^{-\rho\left(E(T) \sigma_{x}\right)},
$$

\footnotetext{
${ }^{3}$ See Appendix 1 for a formal definition of the thickness of a set. ACM Journal Name, Vol. V, No. N, Month 20YY.
} 
where $\rho=\frac{N}{F_{0}}$ denotes the sensor deployment density.

Proof. The proof of Theorem 3 is provided in Appendix 3.

Using Theorem 3, one can select the number of sensors $N$ and the thickness of the sensing areas $E(T)$, in order to guarantee that a target crossing the FoI will be detected before it travels a distance of $\sigma_{x}$ within the FoI. From Theorem 3 we can also evaluate the mean free path that a target $X$ travels undetected.

COROLlary 4. Let $N$ sensors be deployed within the FoI of area $F_{0}$, with each sensor $s_{i}$ having a constant thickness $T_{i}(\theta)=E(T), \forall i$ in all possible directions $\theta$. The mean free path for which a target $X$ remains undetected is:

$$
E(\sigma) \approx \frac{F_{0}}{N E(T)} .
$$

Proof. The proof of Corollary 4 is provided in the Appendix 3.

Note that computing the mean free path $E(\sigma)$ traveled by $X$ is sufficient to determine the mean time $E(t)$ until $X$ is first detected, given a velocity model for $X$. Also note that the constant thickness assumption can by relaxed if one assumes sensing areas of equal thickness on a given direction $\theta$, but not constant over all $\theta$, and then average over all $\theta$.

\section{TARGET DETECTION UNDER DETERMINISTIC SENSOR DEPLOYMENT}

In this section, we compute the target detection probability $P_{D}(1)$ when sensors are deterministically deployed. First, we derive analytical formulas for the deployment of one and two sensors and then generalize for the case where $N$ sensors are deployed. For reasons of notational simplicity we will refer to $P_{D}(1)$ as $P_{D}$.

\subsection{Optimal Placement of a Single Sensor}

Assume that a single sensor $s_{1}$ can be deployed anywhere within the FoI. Based on Theorem 1 the probability $P_{D}$ that a random line $\ell$ intersecting the set $\mathcal{A}_{0}$ $(F o I)$, also intersects the set $\mathcal{A}_{1}$ (sensing area of $s_{1}$ ) can be computed by setting $N=k=1$. In such a case, $P_{D}=\frac{L_{1}}{L_{0}}=\frac{E\left(T_{1}\right)}{E\left(T_{0}\right)}$.

Note that $P_{D}$ is independent of the position of the sensor within the FoI. Hence, placement of the sensor anywhere within the $F o I$ yields the maximum (constant) target detection probability. In figure 2(a), we show the target detection probability $P_{D}$ for varying lengths of the perimeter of sensing area of sensor $s_{1}$, when $s_{1}$ is placed within an FoI of perimeter $200 \pi$. We provide both the theoretical value computed based on Theorem 1, and the simulated value. Figure 2(b) shows the simulated target detection probability when a single sensor is deployed within the $F o I$, as a function of the length of the perimeter of sensor's sensing area. The $P_{D}$ is compared for three differently shaped sensing areas: disk, square and triangle. We observe that the three sensors provide the same target detection capability regardless of the shape of their sensing area due to the identical length of the perimeters. The slight difference among the three curves is an aftermath of the finite resolution used to simulate all possible line trajectories. 


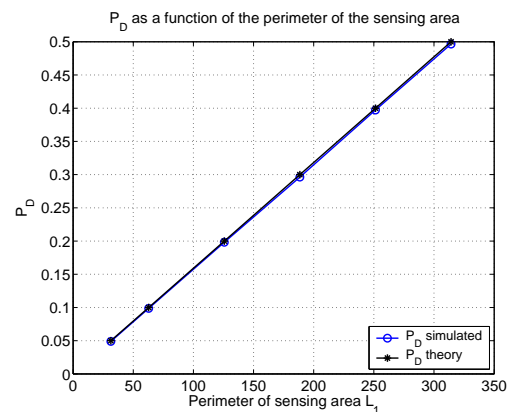

(a)

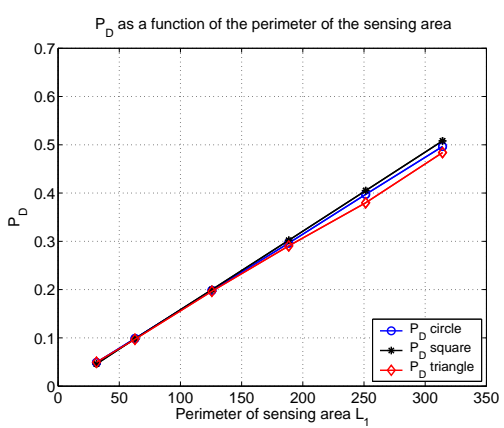

(b)

Fig. 2. (a) The target detection probability $P_{D}$ as a function of the perimeter of the sensing area of a single sensor $s_{1}$. Sensor $s_{1}$ is deployed within a perimeter of $200 \pi$. (b) Comparison of the target detection probability $P_{D}$ achieved by sensors with sensing areas of difference shape but same perimeter, as a function of the length of their perimeter. Regardless of the shape of the sensing areas, the sensors have the same $P_{D}$ for the same length of perimeters.

\subsection{Optimal Sensor Placement of Two Sensors}

Assume that two sensors $s_{i}, s_{j}$ can be placed anywhere within the FoI. The probability of target detection $P_{D}$ can be evaluated based on the following theorem.

THEOREM 4. The target detection probability $P_{D}$ by two sensors $s_{i}, s_{j}$ is:

$$
\begin{gathered}
P_{D}=\frac{L_{i}+L_{j}-m_{2}\left(d_{i, j}\right)}{L_{0}}, \\
m_{2}\left(d_{i, j}\right)=\left\{\begin{array}{cc}
L_{i}+L_{j}-L_{\text {out }}\left(d_{i, j}\right), & \mathcal{A}_{i} \cap \mathcal{A}_{j} \neq \emptyset \\
L_{\text {in }}\left(d_{i, j}\right)-L_{\text {out }}\left(d_{i, j}\right), & \mathcal{A}_{i} \bigcap \mathcal{A}_{j}=\emptyset,
\end{array}\right.
\end{gathered}
$$

where $d_{i, j}$ denotes the pairwise distance between $\mathcal{A}_{i}, \mathcal{A}_{j}, m_{2}\left(d_{i, j}\right)$ denotes the measure of the set of lines intersecting both $\mathcal{A}_{i}, \mathcal{A}_{j}, L_{i n}\left(d_{i, j}\right)$ denotes the length of the inner string wrapped around $\mathcal{A}_{i}, \mathcal{A}_{j}$ as shown in figure 3(b), and $L_{\text {out }}\left(d_{i, j}\right)$ denotes the length of the outer string wrapped around $\mathcal{A}_{i}, \mathcal{A}_{j}$ as shown in figures $3(a), 3(b)$.

Proof. The proof is provided in Appendix 4.

Since the sensing areas are represented as collection of points in the plane, each sensing area $\mathcal{A}$ can be represented with respect to a reference point $O \in \mathcal{A}$. The pairwise distance $d_{i, j}$ between two sets $\mathcal{A}_{i}, \mathcal{A}_{j}$, is measured as the distance among the respective reference points $O_{i}, O_{j}$.

From Theorem 4, we observe that $P_{D}$ is a function of the measure $m_{2}\left(d_{i, j}\right)$ of the set of lines that cross both sensing areas. In the next Lemma, we show that $m_{2}\left(d_{i, j}\right)$ is a monotonically decreasing function of the pairwise distance $d_{i, j}$.

Lemma 1. The measure $m_{2}\left(d_{i, j}\right)$ is a monotonically decreasing function of the pairwise distance $d_{i, j}$ among the sensing areas $\mathcal{A}_{i}, \mathcal{A}_{j}$.

Proof. The proof is provided in Appendix 4.1.

Given the monotonicity of $m_{2}\left(d_{i, j}\right)$, the probability of target detection $P_{D}$ is a monotonically increasing function of $d_{i, j}$. In fact, for circular sensing areas it can be shown that $P_{D}$ tends to an asymptotic value close to $\frac{L_{i}+L_{j}}{L_{0}}$, as depicted in figure ACM Journal Name, Vol. V, No. N, Month 20YY. 


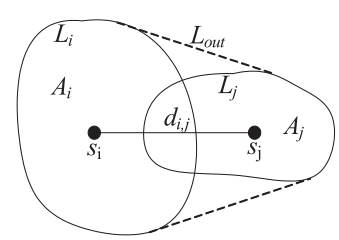

(a)

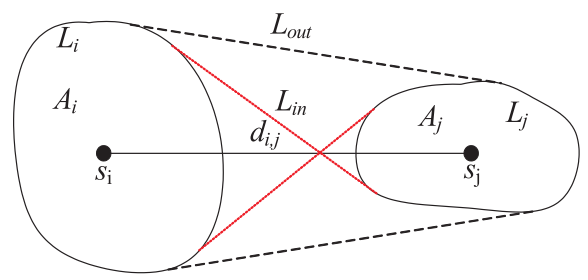

(b)

Fig. 3. The measure $m_{2}\left(d_{i, j}\right)$ of the set of lines intersecting any of the two sensors is equal to (a) $L_{\text {out }}\left(d_{i, j}\right)$ when $\mathcal{A}_{i} \cap \mathcal{A}_{j} \neq \emptyset$, (b) $L_{i}+L_{j}-\left(L_{i n}\left(d_{i, j}\right)-L_{\text {out }}\left(d_{i, j}\right)\right)$ when $\mathcal{A}_{i} \cap \mathcal{A}_{j}=\emptyset$.

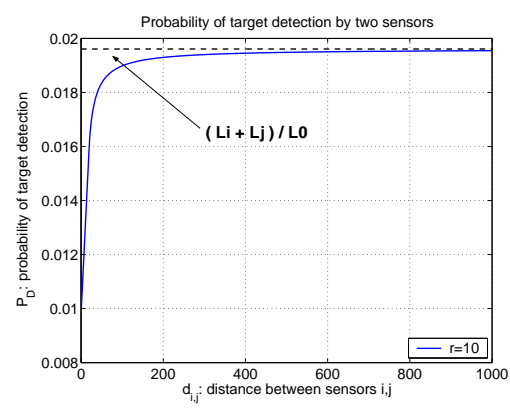

(a)

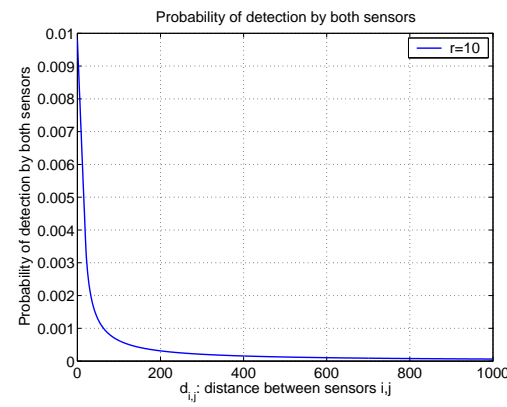

(b)

Fig. 4. The target detection probability $P_{D}$ achieved by two sensors, as a function of the pairwise distance $d_{i, j}$ between the sensors, when each sensors has a circular sensing area of radius $10 \mathrm{~m}$. $P_{D}$ is a monotonically increasing function of $d_{i, j}$ that asymptotically approaches $\frac{L_{1}+L_{2}}{L_{0}}$. (b) The probability that a target is detected by both sensors is a monotonically decreasing function of the pairwise distance $d_{i, j}$, asymptotically approaching zero.

4(a). This is due to the fact that $m_{2}\left(d_{i, j}\right) / L_{0}$, that is, the probability that a line intersects both sets, becomes negligible for $d_{i, j}>>L_{i}, L_{j}$, as illustrated in figure $4(\mathrm{~b})$. Given the monotonicity properties of $m_{2}\left(d_{i, j}\right)$, we can compute the optimal placement of the two sensors that maximizes $P_{D}$.

5.2.1 Sensing areas within the FoI. Assume that the placement of two sensors that yields their respective sensing areas to be at the opposite ends of the diameter of the FoI. Assume also that in such a deployment the entire sensing area of each sensor lays within the FoI, as shown in figure 5(a). Based on the monotonic behavior of $P_{D}$, such placement would result in the maximum target detection probability $P_{D}$ given the $F o I$ boundary, since the pairwise distance $d_{i, j}$ between $\mathcal{A}_{i}, \mathcal{A}_{j}$ attains its maximum value. Hence, the placement that maximizes $P_{D}$, is at the two ends of the diameter of the $F o I$, where $m_{2}\left(d_{i, j}\right)$ is minimized, or alternatively, where the least "number" of lines intersect both sets.

5.2.2 Sensing areas partially within the FoI. Placement of the sensing areas at the two ends of the diameter is optimal if the sensing areas of the sensors fall within the $F o I$, as is the case in figure 5(a). However, since we are considering FoIs of arbitrary shape, it is possible that the sensing areas are only partially laying within the FoI if placed at the diameter, thus overall reducing the sensing capability of 


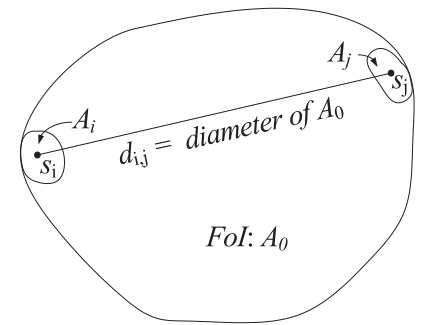

(a)

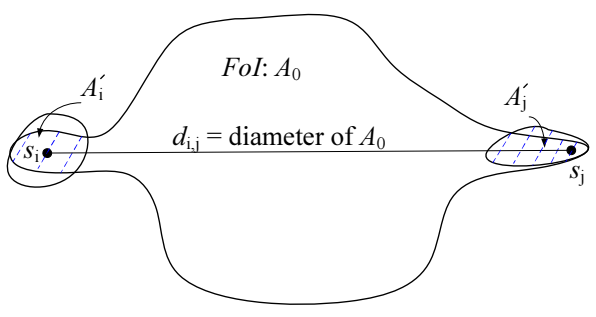

(b)

Fig. 5. Optimal placement of two sensors that maximizes the target detection probability $P_{D}$ in (a) the diameter of a circular FoI, (b) an the diameter of an asymmetric FoI.

the sensors. This is illustrated in figure $5(\mathrm{~b})$, where the $F o I$ becomes very narrow at the two ends of the diameter. Let $\mathcal{A}_{i}^{\prime}=\mathcal{A}_{0} \bigcap \mathcal{A}_{i}$ denote the effective sensing area of $\mathcal{A}_{i}$, that is the sensing area of $s_{i}$ that lays within the FoI. Let also $L_{i}^{\prime}$ denote the effective perimeter of the effective sensing area $L_{i}$.

In such a case the probability of target detection $P_{D}$ is analytically evaluated using Theorem 4 by, (i) substituting the perimeters $L_{i}, L_{j}$ of $\mathcal{A}_{i}, \mathcal{A}_{j}$ with the effective perimeter $L_{i}^{\prime}, L_{j}^{\prime}$ and, (ii) adjusting the lengths of the inner and outer strings $L_{\text {in }}$ and $L_{\text {out }}$ to the lengths of the strings that wrap around sets $\mathcal{A}_{1}^{\prime}, \mathcal{A}_{2}^{\prime}$. Using Theorem 4, and the effective perimeter we can determine the optimal positions of $\mathcal{A}_{1}, \mathcal{A}_{2}$ that yield the maximum $P_{D}$, based on the specific shapes of $\mathcal{A}_{1}, \mathcal{A}_{2}, \mathcal{A}_{0}$.

Note that for an $F o I$ and $\mathcal{A}_{i}, \mathcal{A}_{j}$ of arbitrary shape, $L_{i}^{\prime}, L_{j}^{\prime}, L_{\text {in }}, L_{\text {out }}$ may not have a closed analytical form. Hence, the optimal sensor placement can only be computed numerically. On the other hand, if placement of $\mathcal{A}_{i}, \mathcal{A}_{j}$ in the diameter of the $F o I$ yields $\mathcal{A}_{i}^{\prime}=\mathcal{A}_{i}$ and $\mathcal{A}_{j}^{\prime}=\mathcal{A}_{j}$, then the optimal solution is obtained without any additional knowledge on the shapes of the sets. In most cases it is expected that the placement at the diameter of the $F o I$ will lead to the entire sensing areas being within the FoI due to the assumption that the $F o I$ is significantly larger in dimensions than the sensing areas.

5.2.3 Impact of the sensing area orientation. The $P_{D}$ as expressed by Theorem 4 , is also a function of the sensing area orientation, when the sensing areas are not symmetric. This is due to the fact that the measure $m_{2}\left(d_{i, j}\right)$ is a function of the length of the inner and outer string lengths $L_{i n}, L_{\text {out }}$ which vary with the sensing area orientation, for sensing areas of non-symmetric shapes.

However, in Lemma 1 we showed that $m_{2}\left(d_{i, j}\right)$ is a monotonically decreasing function of the pairwise distance, regardless of the sensing area orientation (see Appendix 4.1). Furthermore, when $d_{i, j}$ is sufficiently large compared to $L_{i}, L_{j}$ the impact of the sensor orientation is minimal. The detection probability $P_{D}$ is maximized when the sensors are placed at the ends of the diameter of the $F o I$ and the sensors are oriented in such a way that $\left(L_{\text {in }}-L_{\text {out }}\right)$ is minimized. The exact sensing area orientations that maximize $P_{D}$ depends on the shape of the sensing area and of the FoI. In the case of arbitrary shapes for $\mathcal{A}_{i}, \mathcal{A}_{j}$, no closed analytic form exists that expresses $\left(L_{\text {in }}-L_{\text {out }}\right)$. Hence, the optimal sensing area orientation can only be obtained via numerical methods (for example via a finite search among quantized orientation positions).

ACM Journal Name, Vol. V, No. N, Month 20YY. 


\subsection{Generalization to the Probability of Detection by $N$ Sensors}

Given $N$ sensors, the target detection probability is analytically expressed by the following theorem.

TheORem 5. Let a target $X$ cross an FoI of perimeter $L_{0}$. Let $N$ sensors be placed within the FoI at any desired position. The target detection probability $P_{D}$ is given by:

$$
\begin{aligned}
P_{D}= & \sum_{i=1}^{N} P\left(\ell \cap \mathcal{A}_{i} \neq \emptyset\right)-\sum_{i, j, i<j} P\left(\ell \cap \mathcal{A}_{i} \cap \mathcal{A}_{j} \neq \emptyset\right) \\
& +\ldots+(-1)^{N+1} P\left(\ell \cap \mathcal{A}_{1} \cap \mathcal{A}_{2} \ldots \cap \mathcal{A}_{N} \neq \emptyset\right) .
\end{aligned}
$$

Proof. The probability that $\operatorname{target} X$ is detected is equivalent to the probability that $X$ crosses the sensing area of at least one sensor. Expressing this statement in terms of probability events:

$$
P_{D}=P\left(\ell \cap \mathcal{A}_{1} \cup \mathcal{A}_{2} \cup \ldots \cup \mathcal{A}_{N}\right) .
$$

By applying the inclusion-exclusion principle [Feller 1971], $P_{D}$ is expressed as the sum of conjunctive probabilities of a line intersecting specific set arrangements.

$$
\begin{aligned}
P_{D}= & P\left(\ell \cap \mathcal{A}_{1} \neq \emptyset\right)+P\left(\ell \cap \mathcal{A}_{2} \neq \emptyset\right) \ldots+P\left(\ell \cap \mathcal{A}_{N} \neq \emptyset\right) \\
& -P\left(\ell \cap \mathcal{A}_{1} \cap \mathcal{A}_{2} \neq \emptyset\right)-P\left(\ell \cap \mathcal{A}_{1} \cap \mathcal{A}_{N} \neq \emptyset\right) \\
& \ldots \\
& +(-1)^{N+1} P\left(\ell \cap \mathcal{A}_{1} \cap \mathcal{A}_{2} \ldots \cap \mathcal{A}_{N} \neq \emptyset\right) \\
= & \sum_{i=1}^{N} P\left(\ell \cap \mathcal{A}_{i} \neq \emptyset\right)-\sum_{i, j, i<j} P\left(\ell \cap \mathcal{A}_{i} \cap \mathcal{A}_{j} \neq \emptyset\right) \\
& +\ldots+(-1)^{N+1} P\left(\ell \cap \mathcal{A}_{1} \cap \mathcal{A}_{2} \ldots \cap \mathcal{A}_{N} \neq \emptyset\right) .
\end{aligned}
$$

While Theorem 5 expresses the exact analytic formula for $P_{D}$, the number of terms in $(12)$ is $\left(2^{N}-1\right)$. Furthermore, for arbitrary set arrangements, analytic expressions of the probability of a line intersecting exactly $k$ sets are not known, except for small values of $k$ [Sylvester 1890]. However, we can compute the following lower and upper bounds for finite unions, that provide worst and best performance guarantees on the target detection probability.

COROLlary 5. The target detection probability $P_{D}$ is bounded by:

$$
\sum_{i=1}^{N} P\left(\ell \cap \mathcal{A}_{i} \neq \emptyset\right)-\sum_{i, j, i<j} P\left(\ell \cap \mathcal{A}_{i} \cap \mathcal{A}_{j} \neq \emptyset\right) \leq P_{D}<\sum_{i=1}^{N} P\left(\ell \cap \mathcal{A}_{i} \neq \emptyset\right) .
$$

Proof. This is a special case of the Bonferroni inequalities [Feller 1971].

Both the lower and upper bound in (14), can be evaluated using Theorems 1, 4:

$$
\frac{1}{L_{0}}\left(\sum_{i=1}^{N} L_{i}-\sum_{i, j, i<j} m_{2}\left(d_{i, j}\right)\right) \leq P_{D}<\frac{1}{L_{0}} \sum_{i=1}^{N} L_{i}
$$


The lower bound in (15) is exact for sensor constellations where no lines intersect more than two sensors. On the other hand, $P_{D}$ can never achieve the upper bound for $N>1$ since there will always be a non-zero measure of lines crossing two sensing areas. The lower bound is a monotonically increasing function of the pairwise distances $d_{i, j}$. This is a consequence of the asymptotic behavior of $P_{D}$ for $N=2$, as shown in Section 5.2, and Appendix 4.1. As the distance $d_{i, j}$ among sensors increase, the measure of the set of lines $m_{2}\left(d_{i, j}\right)$ decreases in a monotonic manner. Since $m_{2}\left(d_{i, j}\right)$ is by definition a positive quantity, the lower bound of $P_{D}$ is a monotonically increasing function of $d_{i, j}$. Hence, by increasing the pairwise distance $d_{i, j}$ among each pair of sensors as much as the boundary of the FoI permits, the lower bound of $P_{D}$ attains its maximum value.

\section{A HEURISTIC ALGORITHM FOR DETERMINISTIC SENSOR PLACEMENT}

In Section 5.3, we showed that the lower bound of $P_{D}$ is a monotonically increasing function of the pairwise distance among the sensors. In this section, we address the problem of finding the WSN constellation that maximizes the lower bound on $P_{D}$ as expressed in (15). Let $C$ denote the WSN constellation obtained after a sensor placement. We want to find the WSN constellation $C^{*}$ such that,

$$
C^{*}=\arg \max _{C}\left(\frac{1}{L_{0}}\left(\sum_{i=1}^{N} L_{i}-\sum_{i, j, i<j} m_{2}\left(d_{i, j}\right)\right)\right),
$$

subject to the constraints imposed on $d_{i, j}$ by the FoI boundary. In (16), the first sum is independent of the WSN constellation $C$. Hence, (16) can be expressed as an equivalent constrained minimization problem,

$$
C^{*}=\arg \min _{C\left(d_{i, j}\right)} \sum_{i, j, i<j} m_{2}\left(d_{i, j}\right),
$$

subject to the constraints imposed on $d_{i, j}$ by the $F o I$ boundary. The problem in (17), is a multivariable constrained optimization problem that may have a closed form solution for specific shapes of $\mathcal{A}_{0}, \mathcal{A}_{i}$. However, for an FoI of arbitrary shape, and for WSN of size larger than a few nodes, the boundary conditions do not have an easy closed analytic form. Hence, we rely on a heuristic placement solution to derive WSN constellations with suboptimum performance. Our solution relies on the analogy of the optimization problem in (17), with the well known problem of finding a 2-dimensional signal constellation that minimizes the average probability of symbol error $P_{S E}$ [Benedetto and Biglieri 1999], and the circle packing problem [Conway and Sloane 1998].

\subsection{Analogy of Target Detection to 2-Dimensional Digital Modulation Schemes}

Assume that an $N$-symbol 2-dimensional signal constellation is used for digital communication over an AWGN channel. For the case where $N=2$, the average symbol error probability $P_{S E}$ is expressed as a function of the pairwise distance $d_{i, j}$ between the two symbols in the plane [Benedetto and Biglieri 1999].

$$
P_{S E}=\frac{1}{2} \sum_{i, i<j} P\left(b_{i} \rightarrow b_{j}\right)=\frac{1}{2} \sum_{i, i<j} \frac{1}{2} \operatorname{erfc}\left(\frac{d_{i, j}}{2 \sqrt{N_{0}}}\right),
$$

ACM Journal Name, Vol. V, No. N, Month 20YY. 
Table I. Analogy of the target detection probability to the symbol error probability.

\begin{tabular}{|ccc|}
\hline Mobile Target Detection & $\leftrightarrow$ & Symbol Error over AWGN \\
\hline Number of sensors $N$ & $\leftrightarrow$ & Number of symbols $N$ \\
Field of Interest $\mathcal{A}_{0}$ & $\leftrightarrow$ & Maximum symbol energy \\
Sensor constellation & $\leftrightarrow$ & Symbol constellation \\
Pairwise distance $d_{i, j}$ & & Pairwise distance $d_{i, j}$ \\
among sensors & $\leftrightarrow$ & among symbols \\
Monotonically decreasing & & Monotonically decreasing \\
function $m_{2}\left(d_{i, j}\right)$ & $\leftrightarrow$ & function $P\left(b_{i} \rightarrow b_{j}\right)$ \\
\hline Maximize the target & & Minimize the average \\
detection probability $P_{D}$ & $\leftrightarrow$ & symbol error probability $P_{S E}$ \\
\hline
\end{tabular}

where $P\left(b_{i} \rightarrow b_{j}\right)$ denotes the probability that a symbol $b_{j}$ has been decoded at the receiver, given that $b_{i}$ was transmitted, erfc denotes the error function, and $\frac{N_{0}}{2}$ denotes the power spectral density of the channel noise component. For fixed maximum signal energy, the symbols are constrained within a disk in the plane. The constraint imposed by the signal energy is analogous to the constraint imposed by the $F o I$ in the WSN problem, when the $F o I$ is circular.

The pairwise symbol error probability $P\left(b_{i} \rightarrow b_{j}\right)$ is a monotonically decreasing function of the pairwise distance $d_{i, j}$ among the symbols [Benedetto and Biglieri 1999], in the same way that $m_{2}\left(d_{i, j}\right)$ is a monotonically decreasing function of the distance $d_{i, j}$ among sensors. For the case of $N=2$, antipodal symbols (symbols placed in the diameter of the disk defined by the maximum energy allowed) minimize $P_{S E}$. This solution is consistent with the optimum sensor placement for $N=2$ sensors, as expressed in Theorem 4.

For the case where $N \geq 2$, the average symbol error probability is expressed in an analogous way as $P_{D}$, using the inclusion-exclusion principle, and can be bounded using the union bound [Benedetto and Biglieri 1999]. For a constellation with $N$ equiprobable symbols, $P_{S E}$ is upper bounded by,

$$
P_{S E} \leq \frac{1}{N} \sum_{i, i<j} P\left(b_{i} \rightarrow b_{j}\right)=\frac{1}{N} \sum_{i, i<j} \frac{1}{2} \operatorname{erfc}\left(\frac{d_{i, j}}{2 \sqrt{N_{0}}}\right) .
$$

Since no analytical form exists to express $P_{S E}$ except for small values of $N$, the digital modulation schemes proposed in the literature [Benedetto and Biglieri 1999], attempt to minimize the upper bound in (19). Minimization of the upper bound in (19), is analogous to the constraint minimization problem as expressed in (17). We want to find the signal constellation $S^{*}$ that minimizes the upper bound in (19),

$$
S^{*}=\arg \min _{S\left(d_{i, j}\right)} \sum_{i, i<j} \operatorname{erfc}\left(\frac{d_{i, j}}{2 \sqrt{N}}\right) .
$$

This problem analogy is presented in Table I. Since the mobile target detection problem as formulated in this article and the minimization of the average symbol error probability over AWGN channels are analogous, signal constellations that perform well in the communication domain, can provide a good heuristic solution for WSN constellations. Thus, our heuristic relies on finding those signal constellations that have good performance in terms of $P_{S E}$. The latter, is related to the circle packing problem in geometry and is investigated in the following section. 


\section{DATE: Deterministic Placement for Target Detection}

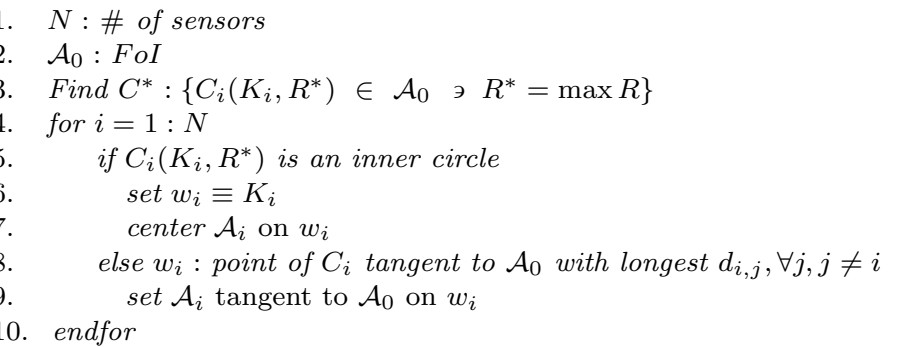

Fig. 6. The pseudocode of DATE.

\subsection{Target Detection and the Circle Packing Problem}

In the digital communications domain, the minimum pairwise distance among symbols is the dominant factor of symbol error, due to the exponential decrease of $P\left(b_{i} \rightarrow b_{j}\right)$ with $d_{i, j}$ [Benedetto and Biglieri 1999]. Hence, good symbol constellations maximize the minimum pairwise distance among symbols. In the WSN domain, $m_{2}\left(d_{i, j}\right)$ decreases proportionally to the inverse of $d_{i, j}$, as shown in figure 4(b). Hence, maximizing the minimum pairwise distance among sensors provides a good heuristic for designing WSN constellations with high $P_{D}$. The problem of maximizing the minimum pairwise distance among points in the plane, can be addressed using the following circle packing problem [Conway and Sloane 1998] as an intermediate step.

Circle Packing Problem: Given $N$ circles $C_{i}, i=1 \ldots N$, compute the maximum radius and positions of the circles that would fit inside a given planar set $\mathcal{A}_{0}$.

The circle packing problem, has known optimal solutions for small values of $N$, $(N \leq 100)$ and certain shapes of $F o I$, such as circle, square, hexagonal or triangle, but no optimal solutions exist for large $N$ [Conway and Sloane 1998]. However, good signal constellations can be carved from lattices with high circle packing density [Conway and Sloane 1998; Boutros et al. 1996; Benedetto and Biglieri 1999]. In the next section, we use the circle packing problem to develop an algorithm for determining WSN constellations with high $P_{D}$.

\subsection{DATE: Deterministic Placement for Target Detection}

In this section, we propose a heuristic algorithm called DATE (Deterministic plAcement for Target dEtection), that is based on the circle packing problem.

Step 1: Find the positions and radius of $N$ circles $C_{i}$, that correspond to the solution of the circle packing problem for $\mathcal{A}_{0}$.

Step 2: For each $C_{i}$, if $C_{i}$ is an inner $\operatorname{circle}^{4}$, choose $w_{i}$ to be the center of $C_{i}$. Else, if $C_{i}$ is an outer circle ${ }^{4}$, choose $w_{i}$ to be the point(s) of $C_{i}$ tangent to $\mathcal{A}_{0}$.

\footnotetext{
${ }^{4} \mathrm{~A}$ circle is called inner if it has no common points with the perimeter of $\mathcal{A}_{0}$, and is called outer if it has at least one common point with $\mathcal{A}_{0}$.
}

ACM Journal Name, Vol. V, No. N, Month 20YY. 


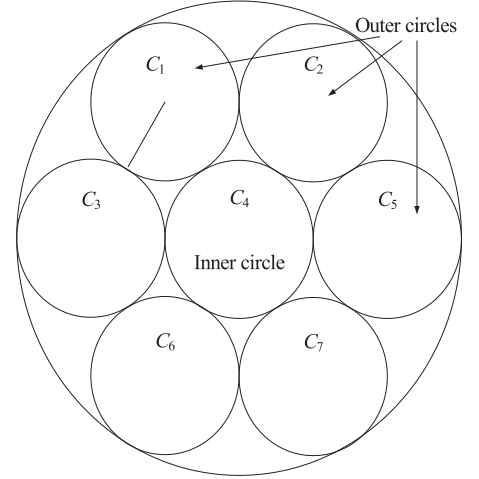

(a)

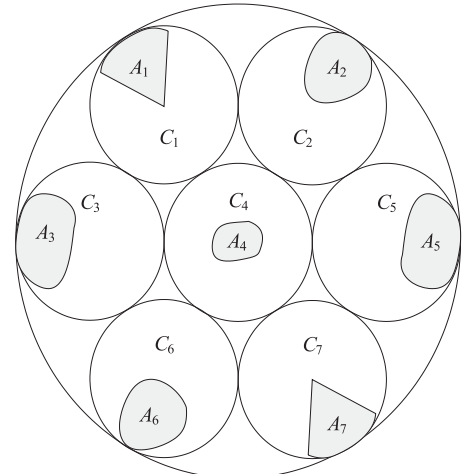

(b)

Fig. 7. The steps of DATE for a WSN of $N=7$ sensors. The shaded circles denote the sensing area of each sensor. (a) The solution to the circle packing problem for $N=7$. Every circle has a radius equal to $\frac{1}{3}$ of the radius of the $F o I$. (b) The placement of the seven sensing areas in the case of heterogeneous WSN. Sensing areas with the longest perimeter, are placed in the diameter of the $F o I$.

Step 3 For each $w_{i}$, if $w_{i}$ is the center of $C_{i}$, center the sensing area of sensor $s_{i}$ on $w_{i}$. Else, if $w_{i}$ is tangent to $\mathcal{A}_{0}$, set the sensing area of sensor $s_{i}$ tangent to $\mathcal{A}_{0}$ on $w_{i}$. If there are more than one tangent points $w_{i}$, choose the tangent point that yields the longest pairwise distance to the rest of the circles.

Note that in Step 3 of our heuristic, we do not take into account the orientation of the sensing areas that are placed inside the FoI. An optimal orientation of the sensing areas can further reduce the $P_{D}$. However, to optimally orient the sensing areas within the circles $C_{i}$, additional information about the exact shape of the sensing areas is required, while DATE only uses the perimeter length of each sensing area. Furthermore, even if the shape of the sensing areas is known, no analytic closed form exist that expresses $P_{D}$ as a function of the sensing area orientation, and hence a numeric solution has to be sought. Finally, when the pairwise distance among the sensing areas is sufficiently large, the impact of the sensing area orientation is minimal, given that $\left(L_{i n}-L_{o u t}\right)$ does not change significantly with the rotation of the sensing areas.

In the case where the sensing areas of the sensors to be placed are heterogeneous, the sensing areas with the longest perimeter are placed at the longest possible pairwise distance apart. By following a greedy approach, the outer circles are initially filled, with the sensing areas of longest perimeter, followed by the inner circles with sensors of smaller sensing areas. Note that our approach guards against sensing area overlap by placing sensing areas with longer perimeter further apart. Sensing area overlap can occur if a sensing area exceeds the dimensions of a circle $C_{i}$. However, this event is not likely, given that $\mathcal{A}_{0}>>\mathcal{A}_{i}, \forall i$, and the number of sensors available is not sufficient to cover the boundary of the FoI.

In figure $7(\mathrm{a})$, we show the solution to the circle packing problem for $N=7$. There are six outer circles and one inner circle, all with a radius equal to $\frac{1}{3}$ of the radius of the $F o I$. In figure $7(\mathrm{~b})$ we show the sensor placement for a WSN with $N=7$ sensors with heterogeneous sensing areas. The sensing areas $\mathcal{A}_{3}, \mathcal{A}_{5}$ (the 


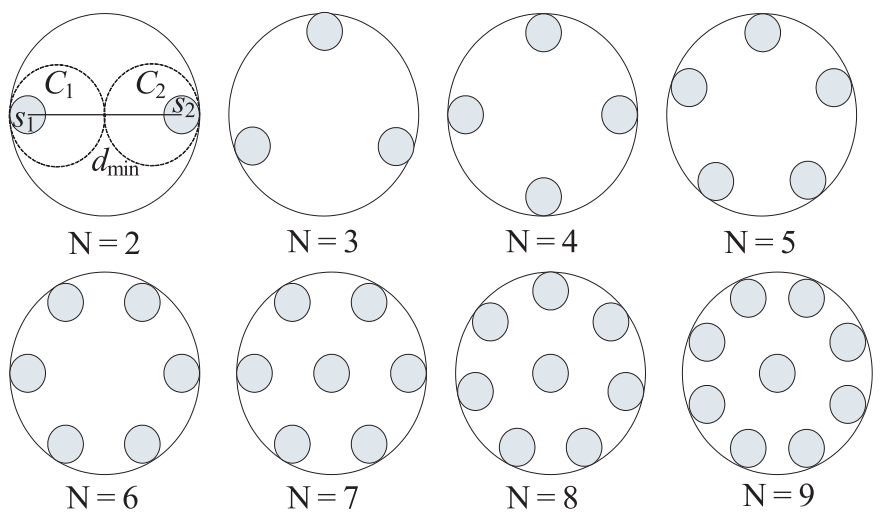

(a)

(b)

Fig. 8. The sensor constellations that maximize the minimum $d_{i, j}$ for a circular FoI.

sensing areas with the longest perimeter), are placed in the diameter of the FoI. Similarly $\mathcal{A}_{1}, \mathcal{A}_{2}, \mathcal{A}_{6}, \mathcal{A}_{7}$ are placed in the diameter of the $F o I$, while $\mathcal{A}_{4}$ is placed in the inner circle, due to its smaller perimeter.

In figure 8(a), we show the optimum placement of sensors in a circular $F_{o I}$, for $N=2 \ldots 9$, according to DATE. Similar solutions exist for FoI of other shapes such as triangles, pentagons, hexagons, or L-shapes [Conway and Sloane 1998].

\section{TARGET DETECTION UNDER THE CONNECTIVITY CONSTRAINT}

Once sensors have detected the presence of a target, they must report the event of detection back to a sink. Time-critical applications such as intrusion detection systems require real-time reporting of the detection event. Hence, a path must exist from every sensor to the sink and the WSN must be connected. For those applications, we consider the target detection problem under the connectivity constraint. First, we provide the definition of a $k$-connected network.

Definition 1. A network is said to be $k$-connected if removal of any $(k-1)$ links does not disconnect the network. Or alternatively, a network is $k$-connected if there are $k$ distinct paths from every node to every other node.

Note that according to the definition of a $k$-connected network, it is not sufficient for each node to have a degree of $k$ to guarantee $k$-connectivity, as it may be possible to have isolated $k$-connected components.

The target detection capability of a WSN and the connectivity constraint establish a tradeoff regarding the placement of the sensors within the FoI. In Section 6 , we showed that increasing the pairwise distance $d_{i, j}$ between sensors increases the target detection probability. On the other hand, to guarantee network connectivity, the pairwise distance among sensors must be reduced so that each sensor has at least a certain number of neighbors. Under the connectivity constraint, the target detection problem is expressed as follows.

Target detection problem under the connectivity constraint: Given an FoI $\mathcal{A}_{0}$ of perimeter $L_{0}$ and $N$ sensors with sensor $s_{i}$ having a sensing area $\mathcal{A}_{i}$ 


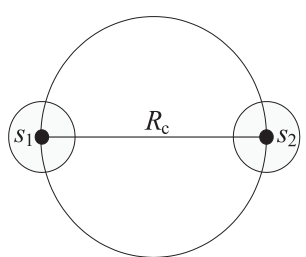

(a)

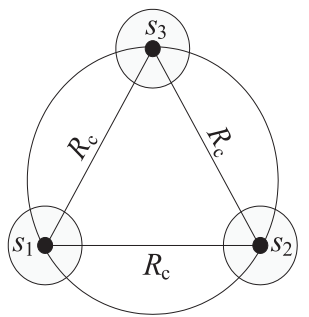

(b)

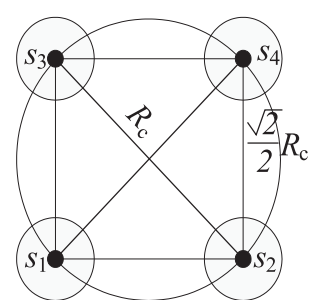

(c)

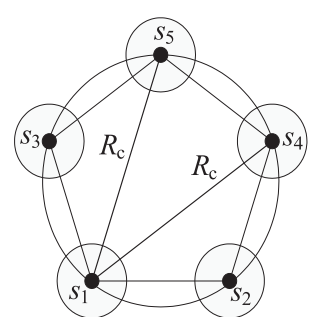

(d)

Fig. 9. The $k$-connected component that maximizes the target detection probability given the connectivity constraint for (a) $k=1$, (b) $k=2$, (c) $k=3$, (d) $k=4$.

of perimeter $L_{i}$, and communication range $R_{i}$, find the WSN constellation that maximizes the probability $P_{D}$, under the condition that the WSN is $k$-connected.

In the problem statement, we assume that each sensor $s_{i}$ has a communication range $R_{i}$, that can differ from sensor to sensor. For clarity purposes we first illustrate our results for the case where sensors have identical communication ranges $R_{c}$ and then generalize to the heterogeneous case.

\subsection{Placement of $(k+1)$ Sensors in a $k$-connected Component}

We first illustrate the placement of $(k+1)$ sensors in a connected component of degree $k$ (every sensor node in the component is connected to $k$ other nodes). The connected component will be used as the building block to generate a $k$-connected network with high target detection probability $P_{D}$.

Assume that $N=k+1$ sensors are available for placement within the FoI. The $(k+1)$ sensors need to be placed within communication range of one another, so that the network is $k$-connected. At the same time we want to maximize the lower bound on the target detection probability as it is expressed in (15), or equivalently, minimize the sum of measures $m_{2}\left(d_{i, j}\right)$, of the set of lines intersecting two sets, as expressed in the minimization problem in (17). The connectivity constraint modifies the boundary conditions in the minimization problem in (17) such that the pairwise distances among all sensors must satisfy: $d_{i, j} \leq R_{c}, \forall i, j i \neq j$.

Following the same design principle as in DATE, we want to find the positions of the sensors so that the minimum pairwise distance is maximized, while at the same time the maximum pairwise distance is less than the communication range $R_{c}$. For maximizing the minimum pairwise distance, the optimal solutions obtained from the circle packing problem can be applied. As an example, in figure 9 we show $k$-connected WSN constellations for $k=1 \ldots 4$. We observe that the $k$-connected WSN constellations are a scaled-down version of the of the solutions of DATE for circular FoI as shown in figure 8(a).

\subsection{Generalization to $k$-connected Networks of $N$ Sensors}

Using the solution for the $k$-connected component, we can generalize to $k$-connected networks of $N$ nodes by expanding the network from a single $k$-connected component. The expansion is done in such a way that every node, or group of nodes added to the network have at least $k$ immediate neighbors. For example assume 


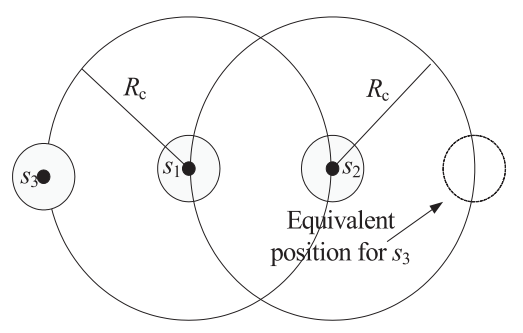

(a)

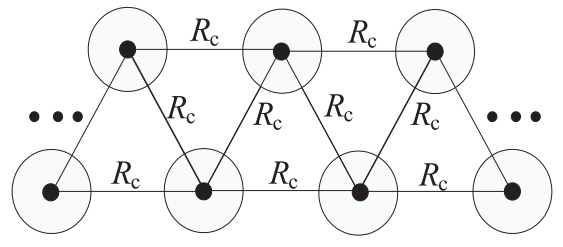

(c)

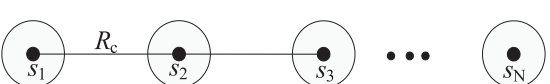

(b)

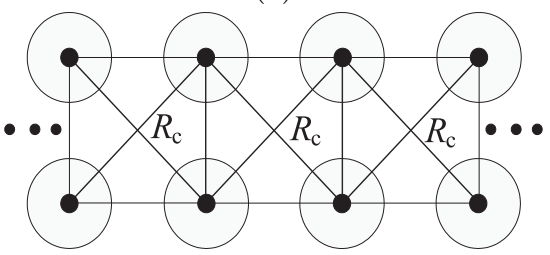

(d)

Fig. 10. (a) The decision space for adding node $s_{3}$ to a 1-connected component, and the two equivalent optimal solutions. (b) A 1-connected network that maximizes the lower bound of $P_{D}$ and satisfies the connectivity constraint. (c) A 2-connected network that maximizes the lower bound of $P_{D}$ and satisfies the connectivity constraint. (d) A 3-connected network that maximizes the lower bound of $P_{D}$ and satisfies the connectivity constraint.

that we want an 1-connected network. The basic component for 1-connectivity is shown in figure $9(\mathrm{a})$, where nodes $s_{1}, s_{2}$ are placed at distance $d_{i, j}=R_{c}$.

When adding a third node $s_{3}$ to the network, $s_{3}$ must be connected to at least one other node so that the network remains 1-connected, and the sum of measures $m_{2}\left(d_{i, j}\right)$ has to be minimized, according to the criterion in (17). Since $m_{2}\left(d_{i, j}\right)$ is a monotonically decreasing function of $d_{i, j}$ we want to select the position for $s_{3}$ that maximizes $d_{1,3}, d_{2,3}$. There are two such candidate positions, as illustrated in figure 10(a), both with the same target detection performance. The rest of the nodes are added in a similar manner forming the serial network shown in figure 10(b).

Similarly, using the basic $k$-connected component, we can construct $k$-connected networks for $k>1$. In figures $10(\mathrm{c}),(\mathrm{d})$, we show the deployment of 2-connected and 3-connected networks, expanded from the 2-connected and 3-connected components, respectively.

\subsection{Accommodating Heterogeneous Communication Ranges}

In the analysis we have done so far, we have assumed that all sensors had the same communication range. This fact can be true even for sensor networks with heterogeneous sensing capabilities, since for communication purposes, the sensors can be equipped with the same radios. However, it is also possible that sensors have heterogeneous communication ranges, due to using sensors from different manufacturers, or deployment of devices with different capabilities. In such a case we can still construct a $k$-connected network using the technique developed for the homogeneous communication range case. Let us first provide the following definition.

Definition 2. Communication range of a $k$-connected component: Let $k+$ 1 nodes be organized in a $k$-connected component, that is, every node is connected to $k$ other nodes. The communication range $R_{g}$ of the $k$-connected component is 


\section{CDATE: Connected DATE}

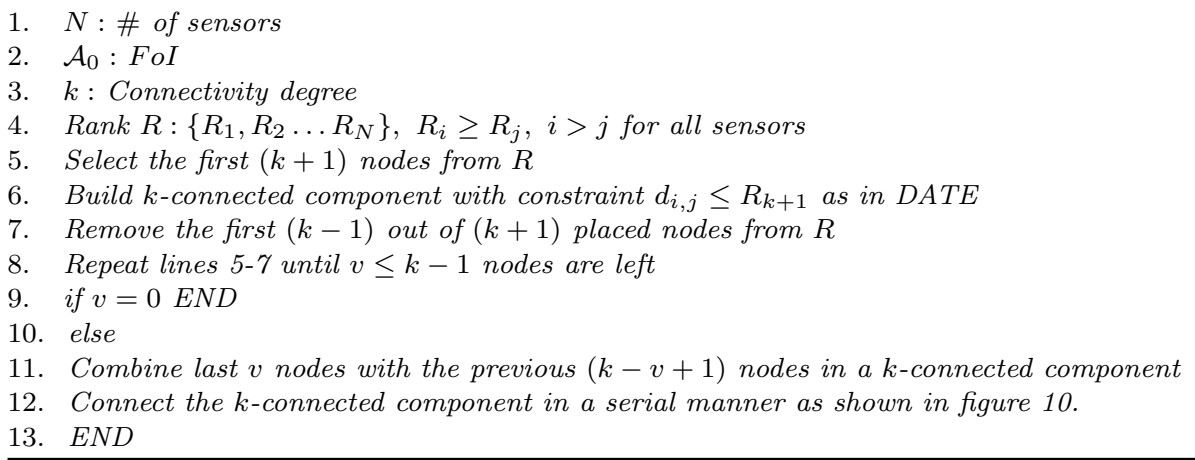

Fig. 11. The pseudocode of CDATE.

defined as the maximum allowable pairwise distance between the $(k+1)$ nodes so that each node has a degree $k$. This distance is equal to the minimum of the communication ranges of the $(k+1)$ sensors,

$$
R_{g}=\min _{i} R_{i}
$$

A $k$-connected network can be constructed using a variant of DATE called CDATE (Connected DATE), illustrated in the following steps:

Step 1: Rank all the sensors $s_{i}, i=1 \ldots N$ to be placed in the $F o I$ in descending order with respect to their communication range,

$$
R:\left\{R_{1}, R_{2}, \ldots R_{N},\right\} R_{i} \geq R_{j} \text {, if } i>j,
$$

where $R_{i}$ denotes the communication range of the sensor ranked $i^{t h}$.

Step 2: Select the first $(k+1)$ nodes and compute the communication range $R_{g}(1)$ of the $k$-connected component.

Step 3: Build the first $k$-connected component with $d_{i, j} \leq R_{g}, \forall i, j i<j$.

Step 4: Expand the $k$-connected component by adding the next $(k-1)$ nodes ${ }^{5}$ at a time, and forming new components. The new component has a communication range $R_{g}(x)$ which is the minimum of the communication ranges of the $(k-1)$ newly added nodes and the last two nodes of the previous component.

Step 5: Repeat Steps 3, 4 until all nodes are added to the network.

Step 6: If there are $(v<k-1)$ residual nodes, the remaining $v$ nodes are combined with $(k-v+1)$ nodes of the previous component.

The pseudocode of CDATE is shown in figure 11. When sensors have heterogeneous communication ranges, our heuristic groups in a $k$-connected component those sensors with the smaller difference in communication ranges (greedy approach). This practice is preferred for increasing the pairwise distances among the sensors in the $k$-connected component. In fact the following can be shown.

${ }^{5}$ For the case of $k=1$, one node is added at the time and not zero as implied by adding $(k-1)$ components at each step. 
THEOREM 6 . The sum $S_{R}$ of the communication ranges $R_{g}(x)$ of the $k$-connected components of a network of $N$ sensors is maximized when the sensors are ranked in descending order and grouped in groups of $(k+1)$ from left to right, as illustrated in Steps 2-6.

Proof. The proof is provided in Appendix 5.

Using CDATE we generate a $k$-connected network where each $k$-connected component has maximum minimum pairwise distance and the connectivity constraint is satisfied. Note that the network has to be constrained within the boundary of the FoI. For example, in the case of 1-connected networks the resulting serial solution will be placed at the diameter of the $F o I$. If the diameter of the $F o I$ is exhausted, the remaining of the nodes are serially deployed along the perimeter of the FoI so as to maximize the pairwise distance among the sensors.

\section{PERFORMANCE EVALUATION}

In this section we validate our theoretical results for the random sensor deployment case and evaluate the performance of our heuristics for the deterministic deployment case. We also illustrate the impact of network parameters such as length of perimeters of sensing areas and communication range (for the network deployment under the connectivity constraint).

\subsection{Random Sensor Deployment}

We randomly deployed $N$ sensors in a circular $F o I$ of radius $R=100 \mathrm{~m}$. We then generated random lines corresponding to random trajectories of targets and measured the number of sensors that detect the moving targets. We repeated our experiments 100 times to ensure statistical validity.

We deployed sensors with heterogeneous sensing capabilities and evaluated the target detection probability. Each sensor deployed had circular sensing area of a radius uniformly distributed in $[0,1]$. We selected a small sensing area in order to satisfy the condition $\max _{i} q_{i} \rightarrow 0$ while $\sum_{i} q_{i} \rightarrow \gamma$, so that the probability of detection of a target by exactly $k$ sensors can be approximated by (7). We varied the number of sensors deployed from $N=100$ to $N=1000$, and computed $P_{D}(1)$.

In figure $12(\mathrm{a})$, we show the theoretical value of $P_{D}(1)$, the value according to Theorem 2, and the simulated value, as a function of $N$. We observe that when the conditions of Theorem 2 are satisfied, one can compute $P_{D}(k)$ without incurring the high computational cost of the exact formula (as $k$ increases the number of terms in the exact formula of $P_{D}(k)$ increase exponentially).

In figure 12(b), we show $P_{D}(1)$ when the radius of the sensors is uniformly distributed in $[0,0.1]$. We observe that the target detection probability grows almost linearly with the number of sensors deployed when the length of the perimeters of the sensing areas of the devices deployed are significantly smaller than the perimeter of the FoI. We note that the Poisson approximation is very close to the exact theoretical value as well as the simulated value.

We also evaluated the probability $P_{M}$ of not detecting a target crossing the sensor field as well as the probability of detection by at least one sensor $P_{D}(1)$, a function of the number of sensors deployed. In figure 12(c) we show $P_{M}$ as a function of $N$. In figure $12(\mathrm{~d})$ we show $P_{D}(1)$ as a function of $N$. From figures $12(\mathrm{c}), 12(\mathrm{~d})$, one can 


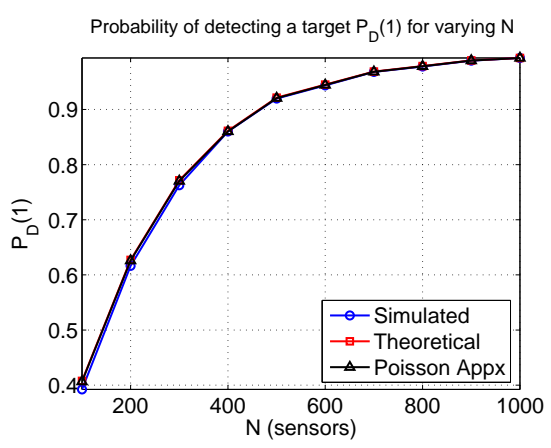

(a)

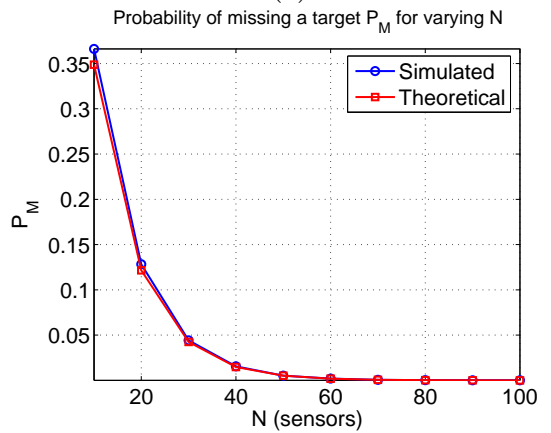

(c)

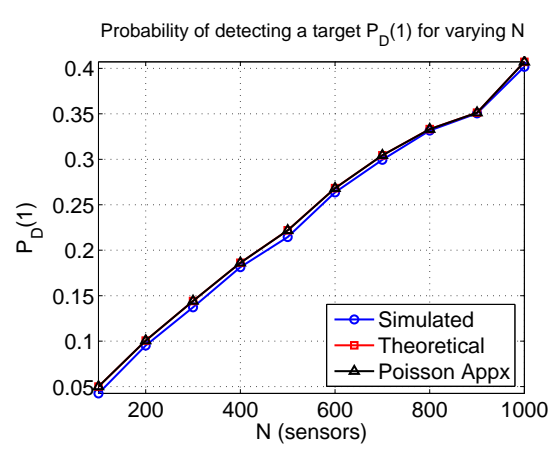

(b)

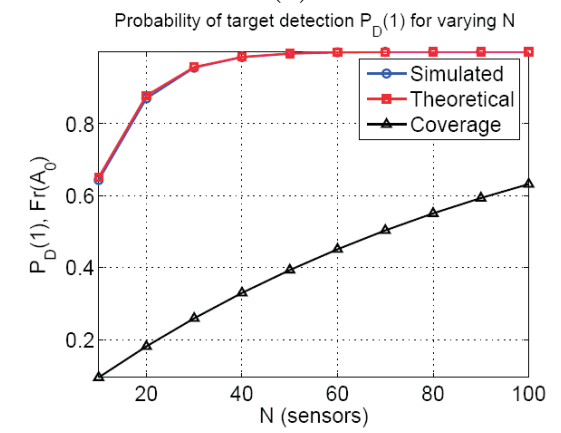

(d)

Fig. 12. $P_{D}(1)$ as a function of $N$, with the radius of $\mathcal{A}$ uniformly distributed in (a) $r \in[0,1]$, (b) $r \in[0,0.1]$. (c) $P_{M}$ as a function of $N$. (d) Comparison of $P_{D}(1)$ with the fraction of the $F o I$ covered by at least one sensor.

select $N$ so that the $P_{D}(1)$ is above a threshold. As an example, if $P_{D}(1) \geq 95 \%$ more than 30 sensors must be deployed.

Figure 12(d), shows the fraction of the $F o I$ denoted as $\operatorname{Fr}\left(\mathcal{A}_{0}\right)$ covered by at least one sensor, as computed using our results for WSN coverage [Lazos and Poovendran 2006]. From figure 12(d), we note that it is not necessary to cover the entire FoI to achieve detection probability close to unity. Thus, target mobility helps in detecting targets with a significantly smaller number of sensors, compared to the number required for detecting static targets.

\subsection{Deterministic Sensor Deployment}

We deployed $N$ sensors within the $F o I$ according to a predefined algorithm such as one of our heuristics or randomly. For each network instance, we generated 10,000 random target trajectories and measured the fraction of trajectories that intersect with the sensing area of one or more sensors. For random deployments, we repeated the experiment for 100 network deployments in order to compute the average target detection probability.

8.2.1 Target Detection Probability for Homogeneous WSN. We placed $N=$ $2 \ldots 9$ sensors in a circular $F o I$ of radius $R d=100 m$, according to the WSN constellations shown in figure 8(a). Sensors had identical sensing ranges that varied from $r=5 m$ to $r=20 \mathrm{~m}$. We measured the target detection probability $P_{D}$ and 


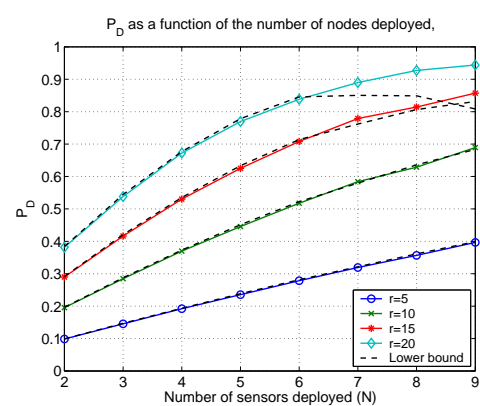

(a)

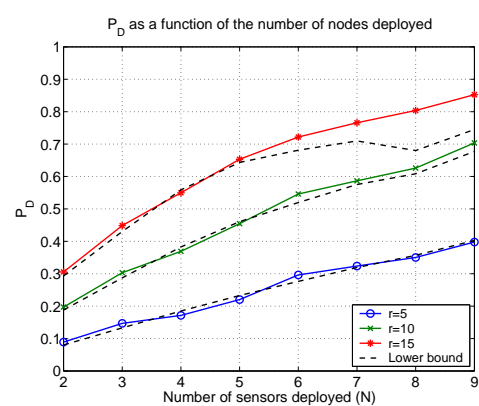

(b)

Fig. 13. The target detection probability $P_{D}$ as a function of the number of sensors deployed and the sensing range radius $r$ for the sensor constellations of, (a) figure 8(a), (b) figure 8(b).

also computed the analytical lower bound given by (15). In figure 13(a), we show the target detection probability $P_{D}$ vs. the number of sensors deployed for varying $r$ and the corresponding lower bound.

We observe that for small values of $r(r=5 m, 10 m)$ the lower bound provides a very good estimate of the actual value of $P_{D}$. This is due to the fact that for the WSN constellations derived by DATE and low values of $r$ relative to $R d$, no lines intersect more than two sensing areas. Hence, the lower bound in (15) that takes into account only lines that intersect one or two sensing areas is exact. Furthermore, we observe that for small values of $r$ the $P_{D}$ increases almost linearly with the number of sensors deployed. This is due to the fact that the measure $m_{2}\left(d_{i, j}\right)$ of lines that intersect two sensing areas is very small when the pairwise distance among the sensors is sufficiently large compared to their sensing range. This is illustrated in figure 3(b) where we show that when $d_{i, j}=20 r$ the probability that a line intersect two sensing areas is almost negligible. Hence, for these values of $r$, the lower bound approaches the upper bound and $P_{D}$ is maximized.

For larger values of sensing range $r$ and WSN values of $N \geq 6$ we observe that the lower bound starts to deviate from the probability of detection $P_{D}$. In fact, the lower bound starts to decrease with the increase of $N$. This is due to the fact that for large values of $r$ and $N$, the probability that a line would intersect three or more sensing areas is non-negligible and hence, omitting this additive factor from the lower bounds yields its deviation from the true value of $P_{D}$.

In figure 13(b), we show the target detection probability $P_{D}$ for WSN constellations deployed within a square with each side being $\alpha=100 \mathrm{~m}$. Again we observe that for small values of $r$ the lower bound holds tightly to the value of $P_{D}$ obtained via the simulation, while the lower bound deviates from $P_{D}$ for large values of $r, N$.

We also studied the performance of DATE for networks of larger size. We considered WSN constellations for $N=\{16,64,128,256\}$, and applied DATE by evaluating well known signal constellations. Specifically we considered constellations drawn from three different lattices, resulting in a circular QAM constellation with 4 amplitude levels, a square QAM constellation, and a checker board QAM constellation [Benedetto and Biglieri 1999]. The constellations for $N=16$ are shown in figure 14, while same shape constellations were considered for higher $N$.

In figure 14 we show the target detection probability as a function of the network size for three different constellations tested. We observe that for $N=\{16,64\}$ 


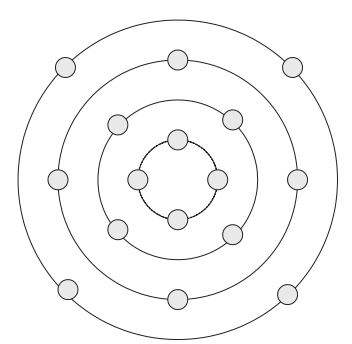

(a)

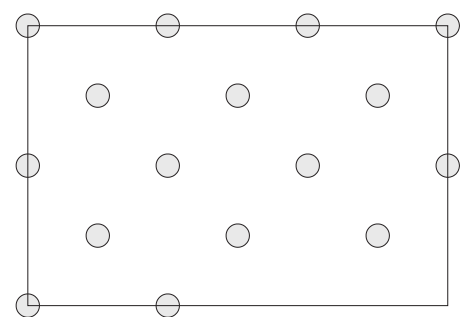

(c)

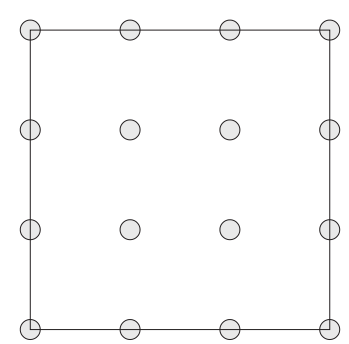

(b)

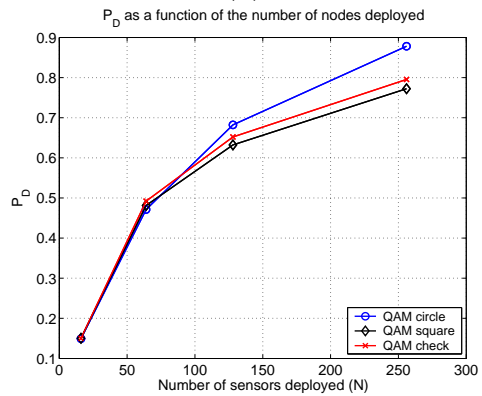

(d)

Fig. 14. Three different QAM constellations used by DATE to generate WSN constellations, for WSN of large size. (a) Circular QAM constellation with 4 amplitude levels. (b) Square QAM constellation (c) Checker board QAM constellation obtained by the square QAM constellation by omitting every other point in a checker board fashion. (d) Comparison of the performance of DATE under the different WSN constellations.

the three constellations have very similar performance in terms of $P_{D}$ while for larger network sizes the circular QAM shows an advantage over the other two constellations. This is mainly due to the fact that we placed the WSN within a circular FoI and hence, the square and checker board QAM do not fully exploit the boundary of the FoI.

We also compared the target detection probability achieved by DATE with the target detection probability achieved by random sensor deployment. Although this comparison is unfair since random deployments yield lower performance due to overlapping sensing areas, it is an indicator of the performance gains that can be achieved by adopting a deterministic solution. For each value of $N$ we randomly deployed the $N$ sensors within the $F o I$ and measured $P_{D}$. We repeated the same experiment 100 times and averaged the result. In figure 15(a), we show the target detection probability for $N=2 \ldots 40$ and for a sensing range $r=5 \mathrm{~m}$. We observe the DATE yields a performance gain up to $14 \%$ compared to random deployment (average case), while random deployment can yield WSN constellations that are up to $90 \%$ worse than the performance of DATE.

In figure $15(\mathrm{~b})$, we show $P_{D}$ for $N=2 \ldots 14$ and for a sensing range $r=20 \mathrm{~m}$. For $r=20 m$ we considered WSN of smaller sizes since larger WSN would be able to entirely cover the boundary of the $F o I$ thus yielding a $P_{D}=1$. We observe that for sensing areas with a larger perimeter, the gains from DATE are even greater, due to the higher sensing area overlap in random deployments. DATE yields a $P_{D}$ up to $18 \%$ higher compared to the performance of the random deployment. 


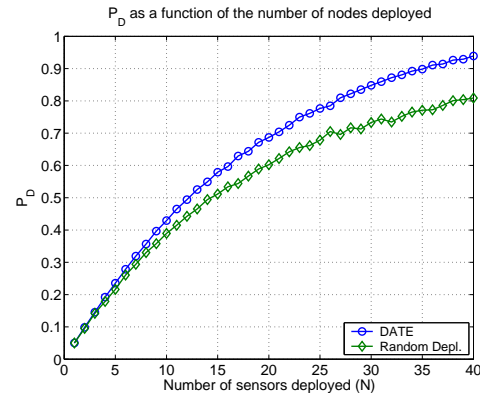

(a)

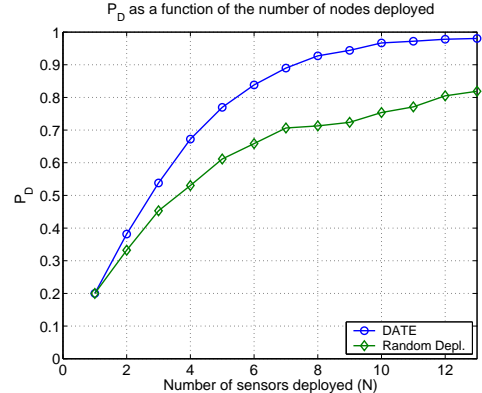

(b)

Fig. 15. Comparison of the performance of DATE with a random deployment strategy for homogeneous WSN with sensing range (a) $r=5 m$, (b) $r=20 m$.

The benefits from adopting DATE compared to a random deployment strategy are even more significant, when the savings in number of sensors is considered. From figure 15(a), we observe that DATE requires 26 sensors to achieve a target detection probability of $P_{D}=0.8$. On the other hand, 40 sensors are required to achieve the same target detection probability using random deployment, that is, $54 \%$ more sensors are required in the random deployment case compared to DATE. Similarly, from figure 15(b), we observe that DATE requires only five sensors to achieve a target detection probability of $P_{D}=0.78$. On the other hand, 11 sensors are required to achieve the same $P_{D}$ using random deployment, that is, $120 \%$ more sensors are required in the random deployment case compared to DATE.

We further evaluated the performance of CDATE by deploying WSN that are $k$-connected. We have assumed that the communication range of each node is four times its sensing range. In figure 16(a), we show the target detection probability for a connected WSN with a varying number of nodes as a function of the degree of connectivity. We observe that the higher the degree of connectivity, the lower the probability of detection. This is due to the fact that a higher degree of connectivity results in a smaller pairwise distance among the sensors.

\subsection{Target Detection Probability for Heterogeneous WSN}

For the case of heterogeneous WSN, we repeated the experiments we conducted for the homogeneous case by placing nodes with heterogeneous sensing areas according to DATE. The shape and size of the sensing areas were randomly selected from a pool of five shapes (circular, square, triangle, pentagon, hexagon). In figures 16(b), 16(c), we show the $P_{D}$ for WSNs of different sizes and as a function of the sensing range $r$. For the heterogeneous WSN case, the sensing range denotes a circle where the sensing area of each sensor can be inscribed. As an example when the sensing area of the selected node is square, the side of the sensing area is equal to $\alpha=\sqrt{(2) r}$, and its perimeter equal to $L_{i}=4 \sqrt{(2)} r$.

We observe that in the heterogeneous case, the lower bound still accurately predicts the target probability of detection when the sensing range is small. For higher values of $r$ the lower bound deviates from $P_{D}$ indicating that a significant number of lines intersect with more than two sensing areas. Also, compared to the homogeneous case, the target detection probability does not exhibit a linear behavior. This is due to the fact that the perimeters of the sensing areas are no longer constant, 


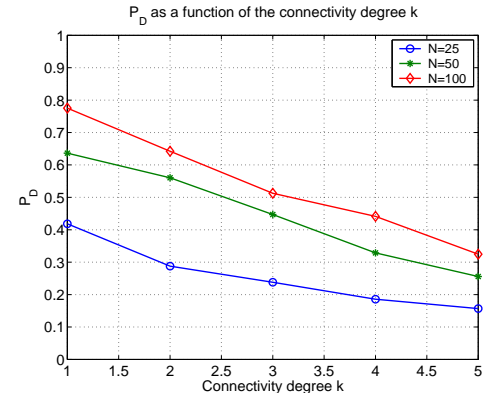

(a)

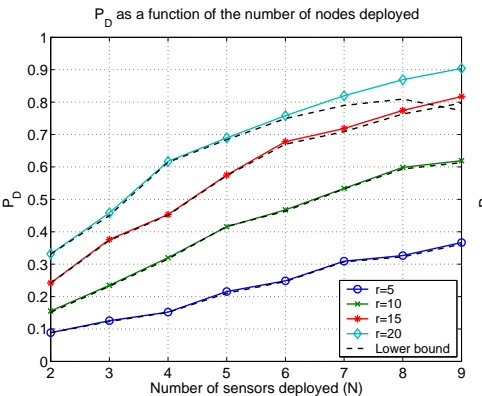

(b)

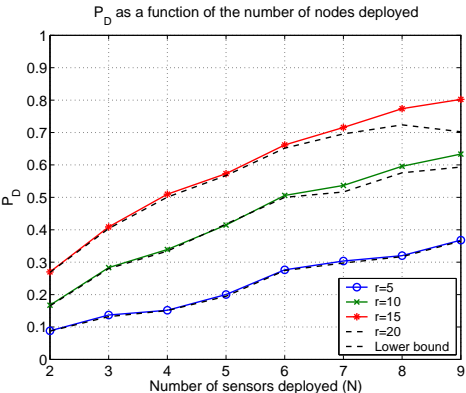

(c)

Fig. 16. The $P_{D}$ obtained by CDATE as a function of (a) the connectivity degree $k$, (b) the number of sensors deployed and the sensing range radius $r$ for circular $F_{o I}$, (c) square FoI. The sensors deployed have heterogeneous sensing capabilities, with the sensing areas randomly selected from a pool of five shapes (circular, square, triangle, pentagon, hexagon). The sensing range denotes a circle where the sensing area can be inscribed.

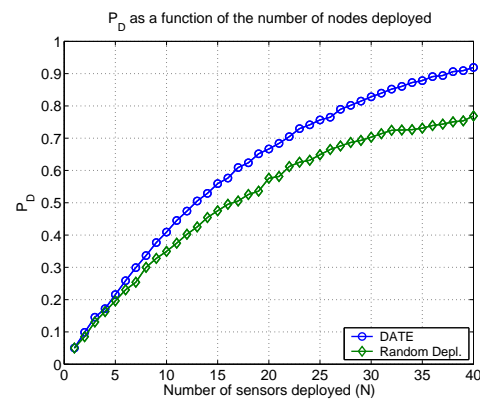

(a)

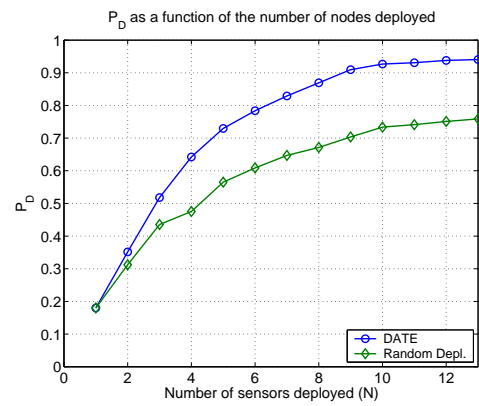

(b)

Fig. 17. Comparison of the performance of DATE with a random deployment strategy for heterogeneous WSN with sensing range (a) $r=5 m$, (b) $r=20 m$.

but vary with the shape of the sensing areas.

We also repeated the comparison of DATE with a random sensor deployment strategy, for heterogeneous WSN. In figure 17, we show the target detection probability as a function of the WSN size. As expected, DATE performs better than the random deployment strategy, with the difference in performance increasing as the number of sensors deployed also increases. Regardless of the shapes of the sensing areas and the lengths of the perimeters, random deployment can result in overlapping sensing areas and sensors with constellations with small pairwise distances, thus having inferior performance to deterministic deployment.

\section{CONCLUSION}

We studied the problem of target detection in heterogeneous WSN. We showed that the target detection problem can be mapped to a line-set intersection problem, and derived analytic formulas that compute the target detection probability. Under random sensor deployment we analytically evaluated the target detection probability $P_{D}(k)$ and the mean free path until a target is detected, and showed that $P_{D}(k)$ is independent of the shape of the sensing areas. Under deterministic deployment, we showed that the complexity of computing the target detection probability $P_{D}$ grows 
exponentially with the WSN size and hence, derived appropriate lower and upper bounds. We further showed that the problem of maximizing the lower bound of $P_{D}$ is analogous to the problem of minimizing the average symbol error probability in 2-dimensional digital modulation schemes. Exploiting this analogy, we proposed a heuristic placement algorithm called DATE, that makes use of well known good signal constellations for determining good WSN constellations. We also considered the problem of maximizing $P_{D}$ under the connectivity constraint, and proposed a heuristic placement algorithm called CDATE.

Our theoretical analysis showed that the principle of maximizing the minimum pairwise distance is a good heuristic solution for the maximization of the worst-case detection probability. Both DATE and CDATE exploit this principle and provide network designers with a systematic way of selecting sensor constellations with probabilistic performance guarantees. This systematic design yields sensor constellations that are not always intuitive and hence, builds a new type of intuition on the problem of target detection based on theoretical analysis rather than empirical evaluations. Finally, both DATE and CDATE incorporate the heterogeneity of different sensing devices in the constellation design in a greedy fashion, leading to better worst-case performance.

\section{APPENDIX}

\section{BACKGROUND ON INTEGRAL GEOMETRY}

A line $\ell(\xi, \theta)$ in the plane, can be represented by the distance $\xi$ of the line from the origin of the coordinate system, and the angle $\theta$ of the line perpendicular to $\ell$, with the $x$ axis. In integral geometry, the measure $m(\ell)$ of a set of lines $\ell(\xi, \theta)$ in the plane is defined as follows [Santaló 2004; Solomon 1978]:

Definition 3. Measure of set of lines $m(\ell)$ : The measure $m$ of a set of lines $\ell(\xi, \theta)$ is defined as the integral over the line density $d \ell=d \xi \wedge d \theta$,

$$
m(\ell)=\int d \xi \wedge d \theta
$$

where $\wedge$ denotes the exterior product used in exterior calculus [Flanders 1989].

In the case where $\mathcal{A}$ is convex, $m(\ell)$ is equal to:

$$
m(\ell: \ell \bigcap \mathcal{A} \neq \emptyset)=\int_{\ell \cap \mathcal{A} \neq \emptyset} d \xi \wedge d \theta=\int_{0}^{2 \pi} \xi d \theta=L,
$$

where $L$ is the perimeter of $\mathcal{A}$. Interested readers are referred to [Santaló 2004; Solomon 1978], for the proof of (24). In the case where $\mathcal{A}$ is non-convex, the measure in (24) can be computed by observing that any line intersecting the convex hull of $\mathcal{A}$, also intersects $\mathcal{A}$. Hence, the measure of the set of lines that intersect a non-convex set is equal to the perimeter of the convex hull of that set. A geometric interpretation for the measure of set of lines as expressed in (24), can be obtained by considering the notion of thickness $T(\theta)$ of a bounded set $\mathcal{A}$ [Solomon 1978].

Definition 4. Thickness of a bounded set $T(\theta)$ : The thickness of a bounded set $\mathcal{A}$ at direction $\theta$ is defined as the length of the projection of $\mathcal{A}$ to a line of direction $\theta$.

ACM Journal Name, Vol. V, No. N, Month 20YY. 


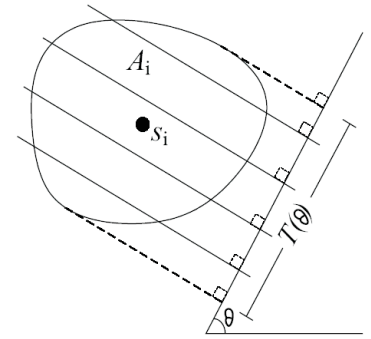

(a)

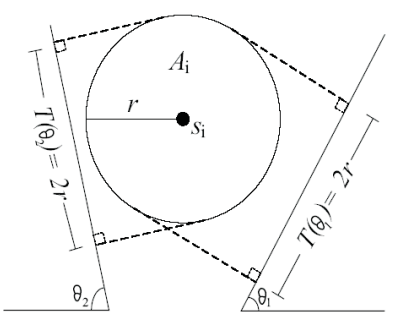

(b)

Fig. 18. (a) The thickness $T(\theta)$ of a set $\mathcal{A}_{i}$ is equal to the length of the projection of $\mathcal{A}_{i}$ on a line with direction $\theta . T(\theta)$ measures the set of lines of direction perpendicular to $\theta$ that intersect $\mathcal{A}_{i}$. (b) For the case of a disk, $T(\theta)=2 r, \forall \theta$, where $r$ is the radius of the disk $\mathcal{A}_{i}$.

The thickness of a set $\mathcal{A}$ measures the set of lines along the direction perpendicular to $\theta$, that intersect $\mathcal{A}$. Integrating the thickness $T(\theta)$ over all possible angles $\theta$ yields the measure of lines crossing the set $\mathcal{A}$. Hence, $T(\theta)$ is related to $m(\ell)$ via:

$$
m(\ell)=\int_{\ell \cap \mathcal{A} \neq \emptyset} d \xi \wedge d \theta \stackrel{(\mathrm{i})}{=} \int_{0}^{\pi} T(\theta) d \theta \stackrel{(\mathrm{ii})}{=} \pi E(T)=L .
$$

Step (i) holds due to the fact that for a fixed $\theta$, the integral of $d \xi$ (set of positions) of the lines that intersect $\mathcal{A}$ is equal to $T(\theta)$. Step (ii) holds due to the random orientation of the lines:

$$
E(T)=\int_{0}^{\pi} \frac{1}{\pi} T(\theta) d \theta
$$

The relation between $m(\ell)$ and $L$ as expressed in (25) can be interpreted as follows. The measure $m(\ell)$ of the set of lines $\ell(\xi, \theta)$ intersecting a bounded set $\mathcal{A}$ is equal to the average length $E(T)$ of the projection of $\mathcal{A}$ over all possible directions, multiplied by the measure of all the possible directions.

Figure 18(a), illustrates the thickness of a set $\mathcal{A}_{i}$ at direction $\theta$. Figure 18(b) illustrates the thickness of a circular sensing area $\mathcal{A}_{i}$, of radius $r$. Independent of the direction of projection, the thickness of a disk is always equal to the diameter of the sensing area, that is $T(\theta)=2 r, \forall \theta$.

\section{PROOF OF THEOREM 1}

ProOF. Let us first compute the probability that a target is detected by a single sensor $s_{i}$. Based on our mapping in Section 3.3, this event is equivalent to the probability $q_{i}$ that a line intersecting $\mathcal{A}_{0}$, also intersects $\mathcal{A}_{i}$. This probability is equal to the quotient of the measure of the set of lines that intersect both $\mathcal{A}_{0}, \mathcal{A}_{i}$ over the measure of the set of lines that intersect $\mathcal{A}_{0}$.

$$
q_{i}=\frac{m\left(\ell \bigcap \mathcal{A}_{0} \bigcap \mathcal{A}_{i} \neq \emptyset\right)}{m\left(\ell \bigcap \mathcal{A}_{0} \neq \emptyset\right)} \stackrel{(\mathrm{i})}{=} \frac{m\left(\ell \bigcap \mathcal{A}_{i} \neq \emptyset\right)}{m\left(\ell \bigcap \mathcal{A}_{0} \neq \emptyset\right)} \stackrel{\text { (ii) }}{=} \frac{L_{i}}{L_{0}} .
$$

Step (i) holds due to the fact that $\mathcal{A}_{i}$ is within $\mathcal{A}_{0}$ and hence, any line intersecting $\mathcal{A}_{i}$ also intersects $\mathcal{A}_{0}$. Step (ii) follows due to (24). The probability $q_{i}$ in (27) is computed for the case where both $\mathcal{A}_{0}, \mathcal{A}_{i}$ are convex sets. In the case where any of the sets are not convex, the length of the perimeter of the convex hull, denoted as $L^{h}$, is used to compute $q_{i}$. 
Using (27), we now compute the probability that a line $\ell$ intersects exactly $k$ sets. Let $Z_{N, k}$ denote the $\left(\begin{array}{c}N \\ k\end{array}\right) k$-tuples $z_{j}$ of vector $[1, \ldots, N]$. That is, $Z_{N, k}=\left\{z_{j}\right.$ : $\left.z_{j(1)}, \ldots, z_{j(i)}, \ldots, z_{j(k)} \mid j(i) \in[1, N], j(i) \neq j(g), \forall i \neq g\right\}$. Let also $\bar{z}_{j}$ denote the complement of $z_{j}$ with respect to the vector $[1, \ldots, N]$. The probability that a line $\ell$ intersects all sets indicated by the $k$-tuple $z_{j}$ is given by:

$$
\begin{aligned}
P\left(z_{j}\right) & \stackrel{\text { (i) }}{=} P\left(\ell \cap \mathcal{A}_{z_{j(1)}} \neq \emptyset, \ldots, \ell \cap \mathcal{A}_{z_{j(k)}} \neq \emptyset, \ell \cap \mathcal{A}_{\bar{z}_{j(1)}}=\emptyset, \ldots, \ell \cap \mathcal{A}_{\bar{z}_{j(N-k)}}=\emptyset\right) \\
& \stackrel{(\mathrm{ii})}{=} P\left(\ell \cap \mathcal{A}_{z_{j(1)}} \neq \emptyset\right) \ldots P\left(\ell \cap \mathcal{A}_{z_{j(k)}} \neq \emptyset\right) \ldots P\left(\ldots \ell \cap \mathcal{A}_{\bar{z}_{j(N-k)}}=\emptyset\right) \\
& =\prod_{i=1}^{\left|z_{j}\right|} q_{z_{j}(i)} \prod_{v=1}^{|\bar{z} j|}\left(1-q_{\bar{z}_{j}(v)}\right) .
\end{aligned}
$$

In Step (i), we express $P\left(z_{j}\right)$ as the probability that a random line intersects exactly the $k$ sets denoted by the $k$-tuple $z_{j}$. Since the sets $\mathcal{A}_{i}$ are randomly and independently deployed within the FoI, in Step (ii) the probability of the intersection of events becomes equal to the product of the probabilities of the individual events. To compute the probability of a random line intersecting any $k$ sets, $P\left(z_{j}\right)$ must be summed over all possible $k$-tuples $z_{j}$.

$$
P\left(Z_{N, k}\right)=\sum_{Z_{N, k}} \prod_{i=1}^{\left|z_{j}\right|} q_{z_{j}(i)} \prod_{v=1}^{|\bar{z} j|}\left(1-q_{\bar{z}_{j}(v)}\right) .
$$

Theorem 1 holds by noting that $P_{D}(k)=1-\sum_{w=0}^{k-1} P\left(Z_{N, w}\right)$.

\section{PROOF OF THEOREM 3}

Proof. A target $X$ travels for a distance $\sigma_{x}$ undetected, if it does not cross the sensing area of any of the deployed sensors. When the sensors have a sensing area of identical thickness for all $\theta$, any sensor within $\frac{E(T)}{2}$ from the trajectory of $X$, detects $X$. This is equivalent to considering that the target $X$ has constant thickness $E(T)=T(\theta), \forall \theta$, while the sensing area of all sensors is reduced to point masses. Figure 19(a) illustrates target $X$ being detected by sensors with sensing areas of constant thickness $E(T)=$ constant. Figure 19(b) illustrates the equivalent scenario, where the target $X$ has constant thickness, while the sensing areas are reduced to point masses.

When the target $X$ moves a distance $\sigma_{X}$, it covers an area of size $\mathcal{F}\left(\sigma_{X}\right)=$ $E(T) \sigma_{x}+f$, where $f$ denotes the residual shaded area in figure 19(c). Hence, the probability that the target $X$ is not detected for a distance of $\sigma \geq \sigma_{x}$ is equal to the probability that no sensor is located within $\mathcal{F}\left(\sigma_{x}\right)$. Given the random sensor deployment, the number of sensors within $\mathcal{F}\left(\sigma_{x}\right)$ is given by a homogeneous planar Poisson point process of density $\rho=\frac{N}{F_{0}}$ [Santaló 2004]:

$$
P(|S|=k)=\frac{(\rho F)^{k}}{k !} e^{-\rho F},
$$

where $|S|$ denotes number of sensors. Equation (30) holds under the assumption that $F_{0} \rightarrow \infty$, while the sensor density $\rho$ remains constant. Based on (30), the probability that the free path of $\operatorname{target} X$ is $\sigma \geq \sigma_{x}$, is equal to the probability that 


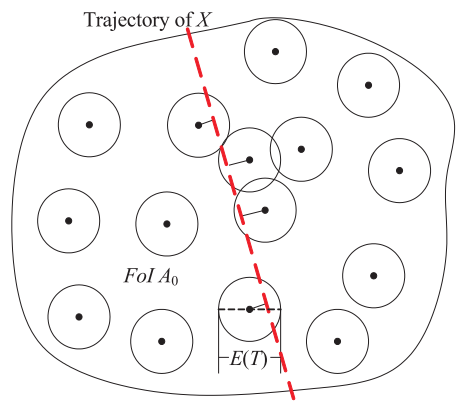

(a)

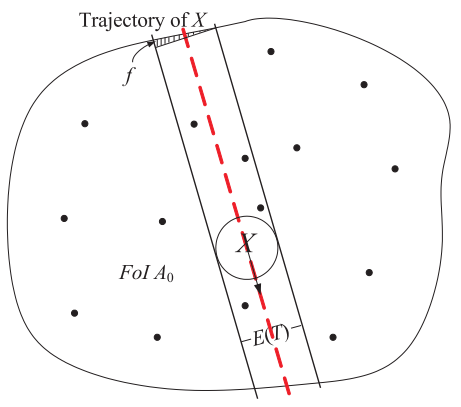

(b)

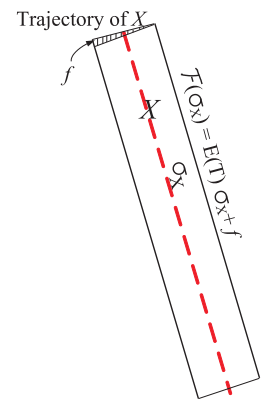

(c)

Fig. 19. (a) Any sensor within a distance $\frac{E(T)}{2}$ from the trajectory of the target $X$, detects X. (b) Equivalent formulation for a target of average thickness $E(T)$, and sensors with sensing areas reduced to point masses (c) The mean free path of a target $X$ and the equivalent free area.

no sensors exist within an area of size $\mathcal{F}\left(\sigma_{x}\right)$ :

$$
P\left(\sigma \geq \sigma_{x}\right)=P\left(N_{\mathcal{F}\left(\sigma_{x}\right)}=0\right)=e^{-\rho \mathcal{F}\left(\sigma_{x}\right)}=e^{-\rho\left(E(T) \sigma_{x}+f\right)} \approx e^{-\rho\left(E(T) \sigma_{x}\right)},
$$

assuming that the residual area is small enough such that $e^{-\rho f} \approx 1$.

\subsection{Proof of Corollary 4}

Proof. The random variable $\sigma$ expressing the free path is a non-negative continuous random value and, hence its expectation is given by:

$$
E(\sigma)=\int_{0}^{Q} P\left(\sigma \geq \sigma_{x}\right) d \sigma_{x}=\frac{e^{-\rho f}}{\rho E(T)}\left(1-e^{-\rho E(T) Q}\right),
$$

where $Q$ denotes the maximum possible length of the trajectory of $X$ within the FoI. When the residual area $f$ is small enough so that $e^{-\rho f} \approx 1$ and $Q$ is long enough so that $e^{-\rho E(T) Q} \approx 0$,

$$
E(\sigma) \approx \frac{1}{\rho E(T)}=\frac{F_{0}}{N E(T)}
$$

\section{PROOF OF THEOREM 4}

Proof. For the case of two sensors $s_{i}, s_{j}$, a mobile target $X$ is detected if its trajectory crosses the sensing area of either $s_{i}$ or $s_{j}$. Using the equivalence to the line-set intersection problem, the target detection probability $P_{D}$ is the probability that a random line intersects any of the two sets $\mathcal{A}_{i}, \mathcal{A}_{j}$ placed within the $F_{o I}$, $\mathcal{A}_{0}$. Expressed in probability terms,

$$
P_{D}=P\left(\ell \cap \mathcal{A}_{i}\right)+P\left(\ell \cap \mathcal{A}_{j}\right)-P\left(\ell \cap \mathcal{A}_{i} \cap \mathcal{A}_{j}\right) \stackrel{(i)}{=} \frac{L_{i}+L_{j}-m_{2}\left(d_{i, j}\right)}{L_{0}} .
$$

In Step (i), $P\left(\ell \cap \mathcal{A}_{i}\right), P\left(\ell \cap \mathcal{A}_{j}\right)$ are computed using Theorem 1 , and are independent of the positions of the two sets $\mathcal{A}_{i} \mathcal{A}_{j}$. However, the measure $m_{2}\left(d_{i, j}\right)$ of the set of lines intersecting both $\mathcal{A}_{i} \mathcal{A}_{j}$, is a function of the relative distance $d_{i, j}$ between $\mathcal{A}_{i}, \mathcal{A}_{j}$, and is computed based on the following two cases. 
Case I $-\mathcal{A}_{i} \cap \mathcal{A}_{j} \neq \emptyset$ : When the sensing areas $\mathcal{A}_{i}, \mathcal{A}_{j}$ overlap, as shown in figure 3(a), $\mathcal{A}_{i}, \mathcal{A}_{j}$ form a connected and bounded set $\mathcal{A}_{c}=\mathcal{A}_{i} \cup \mathcal{A}_{j}$, and the target $X$ is detected if it crosses $\mathcal{A}_{c}$. According to (24), the measure of the set of lines intersecting the bounded and connected set $\mathcal{A}_{c}$ is equal to the perimeter of $\mathcal{A}_{c}$, when $\mathcal{A}_{c}$ is convex, or the perimeter of the convex hull of $\mathcal{A}_{c}$ when $\mathcal{A}_{c}$ is not convex (when $\mathcal{A}_{c}$ is convex, $\mathcal{A}_{c}$ is the convex hull of itself by definition).

For two sets intersecting, the convex hull can be found by wrapping a string around the two sets, as shown in figure 3(a). Any line intersecting with the convex hull of $\mathcal{A}_{c}$, is guaranteed to intersect with at least one of $\mathcal{A}_{i}, \mathcal{A}_{j}$. Using Theorem 1 , the target detection probability by two sensors with intersecting sensing areas is equal to:

$$
P_{D}=\frac{L_{i}+L_{j}-m_{2}\left(d_{i, j}\right)}{L_{0}}=\frac{L_{\text {out }}\left(d_{i, j}\right)}{L_{0}}, \quad \mathcal{A}_{i} \bigcap \mathcal{A}_{j} \neq \emptyset,
$$

where $L_{\text {out }}\left(d_{i, j}\right)$ denotes the length of the perimeter of the convex hull of $\mathcal{A}_{c}$ (outer string in figure 3(a)). From (35), the measure of the set of lines intersecting both $\mathcal{A}_{i}, \mathcal{A}_{j}$ is, $m_{2}\left(d_{i, j}\right)=L_{i}+L_{j}-L_{\text {out }}\left(d_{i, j}\right)$.

Case II $-\mathcal{A}_{i} \cap \mathcal{A}_{j}=\emptyset:$ When the sensing areas $\mathcal{A}_{i}, \mathcal{A}_{j}$ do not overlap, as shown in figure $3(\mathrm{~b}), \mathcal{A}_{i}, \mathcal{A}_{j}$ no longer form a connected and bounded set. Sylvester showed that the measure of all lines that intersect both $\mathcal{A}_{i}, \mathcal{A}_{j}$ is equal to $m_{2}\left(d_{i, j}\right)=$ $L_{\text {in }}\left(d_{i, j}\right)-L_{\text {out }}\left(d_{i, j}\right)$ [Sylvester 1890]. Hence in the case of non-overlapping $\mathcal{A}_{i}, \mathcal{A}_{j}$, $P_{D}$ is equal to:

$$
\begin{aligned}
P_{D} & =\frac{L_{i}+L_{j}-m_{2}\left(d_{i, j}\right)}{L_{0}} \\
& =\frac{L_{i}+L_{j}-\left(L_{i n}\left(d_{i, j}\right)-L_{\text {out }}\left(d_{i, j}\right)\right)}{L_{0}}, \mathcal{A}_{i} \bigcap \mathcal{A}_{j}=\emptyset .
\end{aligned}
$$

\subsection{Proof of Monotonicity of $m_{2}\left(d_{i, j}\right)$}

Proof. To prove the monotonicity of $m_{2}\left(d_{i, j}\right)$, we must show that $m_{2}\left(d_{i, j}\right)$ becomes smaller as the distance $d_{i, j}$ among the sensing area $\mathcal{A}_{i}, \mathcal{A}_{j}$ increases. For cases where $L_{\text {in }}, L_{\text {out }}$ have an analytical form, this can be very easily shown by computing the first derivative of $\left(L_{i n}-L_{\text {out }}\right)$, with respect to $d_{i, j}$. As an example, when the two sensing areas are discs of radius $r$, we can analytically express $L_{i n}, L_{\text {out }}$ as follows:

$$
\begin{aligned}
L_{\text {in }} & =2\left(\pi r+\arctan \left(\frac{2 r}{d_{i, j}}\right) r+2 \sqrt{\frac{d_{i, j}^{2}}{4}-r^{2}}\right), \\
L_{\text {out }} & =2\left(\pi r+d_{i, j}\right) .
\end{aligned}
$$

Computation of the derivative of $\left(L_{i n}-L_{\text {out }}\right)$ verifies the monotonicity of $m_{2}\left(d_{i, j}\right)$ (this is an elementary mathematic exercise not presented here due to space constraints). For the case where $\left(L_{i n}-L_{\text {out }}\right)$ does not have a closed analytic form, we can illustrate the monotonicity of $m_{2}\left(d_{i, j}\right)$ by considering its equivalence to the set of lines intersecting both sets. The argument in our proof is that as the distance

ACM Journal Name, Vol. V, No. N, Month 20YY. 


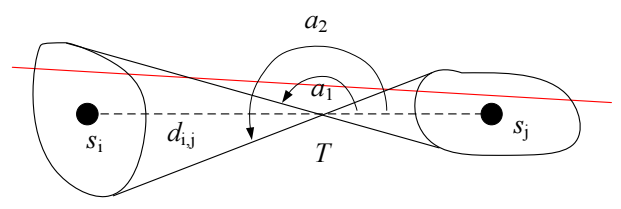

(a)

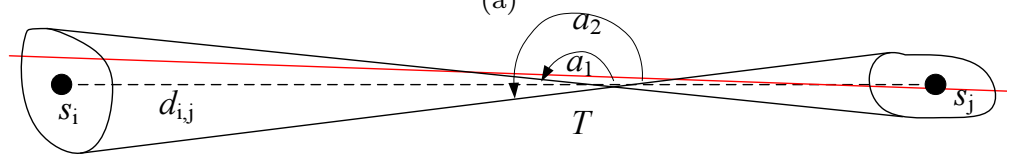

(b)

Fig. 20. (a) Any line intersecting both sensing areas has a slope $a_{1} \leq a \leq a_{2}$, (b) when $d_{i, j}$ increases, the slope difference $a_{2}-a_{1}$ decreases and, hence, a smaller number of lines intersects both sensing areas. Therefore, the measure $m_{2}\left(d_{i, j}\right)$ of the set of lines intersecting two sets is a monotonically decreasing function of $d_{i, j}$.

between the sensing areas increases, a smaller "number" of lines will intersect both sets and, hence, $m_{2}\left(d_{i, j}\right)$ becomes smaller.

Let $a_{1}, a_{2}$ denote the slopes of the lines of the inner string that wraps around $\mathcal{A}_{i}, \mathcal{A}_{j}$, as shown in figure 20(a). Any line that is crossing both $\mathcal{A}_{i}, \mathcal{A}_{j}$ must have a slope $a$ with $a_{1} \leq a \leq a_{2}$. As an example, all lines that pass through the intersection point $T$ with slope $a_{1} \leq a \leq a_{2}$ intersect both sets. The measure of the set of lines crossing both sets is monotonically related to the range of $\left(a_{2}-a_{1}\right)$ that is the greater the difference between the slopes $a_{1}, a_{2}$ the larger the "number" (measure) of lines that cross both sets (more lines out of all possible trajectories satisfy the $a_{1} \leq a \leq a_{2}$ condition).

As the distance $d_{i, j}$ between the sets $\mathcal{A}_{i}, \mathcal{A}_{j}$ increases, the slope difference $\left(a_{2}-a_{1}\right)$ decreases and, hence the "number" of lines intersecting both sets also decreases. Therefore, $m_{2}\left(d_{i, j}\right)$ which expresses the measure of the set of lines intersecting both sets, also decreases with the decrease of the slope difference, or equivalently with the increase of the pairwise distance $d_{i, j}$. In figure $20(\mathrm{~b})$, we show the reduction in the slope difference $\left(a_{2}-a_{1}\right)$ that leads to the reduction of the set of lines that intersect both sets.

\section{PROOF OF THEOREM 6}

Proof. Let the communication ranges of the $N$ sensors be ranked in descending order as in Step 1. Let also $m$ groups of $k+1$ nodes be generated by selecting $k+1$ nodes at a time from left to right with each group having two overlapping nodes with the previous group (for connectivity purposes), as shown in Steps 2-6. Then the sum $S_{R}$ of the communication ranges of the $m k$-connected components is:

$$
S_{R}=\sum_{x} R_{g}(x)
$$

Assume that two the communication ranges of two sensors $R_{i}, R_{j}, i>j$ are swapped in the rank ordering. If $i, j$ belong to the same $k$-connected component, $S_{R}$ does not change since the communication range of all components remains the same.

Assume now that the two communication ranges belong to two different $k$ - 
connected components $x_{1}, x_{2}$ (the two swapped sensors belong to different groups). Let the two components have communication ranges of $R_{g}\left(x_{1}\right), R_{g}\left(x_{2}\right)$ before the swap, respectively, and communication ranges $R_{g}^{\prime}\left(x_{1}\right), R_{g}^{\prime}\left(x_{2}\right)$ after the swap, respectively. The difference between the old sum $S_{R}$ and the new sum $S_{R}^{\prime}$ is,

$$
S_{R}-S_{R}^{\prime}=\left(R_{g}\left(x_{1}\right)-R_{g}^{\prime}\left(x_{1}\right)\right)+\left(R_{g}\left(x_{2}\right)-R_{g}^{\prime}\left(x_{2}\right)\right) .
$$

Since $i, j$ belong to different components with $x_{1}, x_{2}$ and $i>j$ this implies that

$$
R_{g}\left(x_{1}\right)>R_{g}\left(x_{2}\right),
$$

and every element of $x_{1}$ has a communication range greater or equal of every element of $x_{2}$. Hence, $R_{g}\left(x_{1}\right) \geq R_{j}$ and after the swap,

$$
R_{g}^{\prime}\left(x_{1}\right)=R_{j}
$$

Therefore, $\left(R_{g}\left(x_{1}\right)-R_{g}^{\prime}\left(x_{1}\right)\right) \geq 0$. Furthermore, if $R_{j}$ is not the smallest communication range among the $(k+1)$ communication ranges that belong to $x_{2}$ the communication range of the $k$-component $x_{2}$ after the swap will not change,

$$
R_{g}\left(x_{2}\right)=R_{g}^{\prime}\left(x_{2}\right) .
$$

In this case $S_{R}-S_{R}^{\prime} \geq 0$ and the swap of the two elements cannot increase the sum $S_{R}$. Since this holds true for any swap, the method described in Theorem 6 yields the highest sum of communication ranges of the $k$-connected components.

If $R_{j}$ is the smallest component in $x_{2}$, then $R_{j}=R_{g}\left(x_{2}\right)$. But also swapping $R_{j}$ in component $x_{1}$ will yield according to (42),

$$
R_{g}^{\prime}\left(x_{1}\right)=R_{j}=R_{g}\left(x_{2}\right) .
$$

At the same time swapping $R_{i}$ to $x_{2}$ yields $R_{g}^{\prime}\left(x_{2}\right) \leq R_{g}\left(x_{1}\right)$ since every element in $x_{2}$ is less than every element in $x_{1}$, hence also equal or less than the minimum of $x_{1}$. A substitution in (40) yields,

$$
\begin{aligned}
S_{R}-S_{R}^{\prime} & =\left(R_{g}\left(x_{1}\right)-R_{g}^{\prime}\left(x_{1}\right)\right)+\left(R_{g}\left(x_{2}\right)-R_{g}^{\prime}\left(x_{2}\right)\right) \\
& =R_{g}\left(x_{1}\right)-R_{g}\left(x_{2}\right)+R_{g}\left(x_{2}\right)-R_{g}^{\prime}\left(x_{2}\right) \\
& =R_{g}\left(x_{1}\right)-R_{g}^{\prime}\left(x_{2}\right) \geq 0 .
\end{aligned}
$$

\section{REFERENCES}

Aslam, J., Butler, Z., Constantin, F., Crespi, V., Cybenko, G., And Rus, D. 2003. Tracking a moving object with a binary sensor network. Proceedings of the First International Conference on Embedded Networked Sensor Systems, 150-161.

Benedetto, S. And Biglieri, E. 1999. Principles of Digital Transmission: With Wireless Applications. Kluwer Academic Publishers.

Boutros, J., Viterbo, E., Rastello, C., And Belfiore, J. 1996. Good lattice constellations for both Rayleigh fading and Gaussian channels. Information Theory, IEEE Transactions on 42, 2, 502-518.

Cao, Q., Yan, T., Stankovic, J., and Abdelzaher, T. 2005. Analysis of target detection performance for wireless sensor networks. DCOSSO5.

Сно, E. 2002. Grid Coverage for Surveillance and Target Location in Distributed Sensor Networks. IEEE Transactions On Computers 51, 12, 1449.

ACM Journal Name, Vol. V, No. N, Month 20YY. 
Clouqueur, T., Phipatanasuphorn, V., Ramanathan, P., and Saluja, K. 2002. Sensor deployment strategy for target detection. Proceedings of the 1st ACM International Workshop on Wireless Sensor Networks and Applications, 42-48.

Clouqueur, T., Saluja, K., And Ramanathan, P. 2004. Fault tolerance in collaborative sensor networks for target detection. IEEE Transactions on Computers 53, 3, 320-333.

Conway, J. And Sloane, N. 1998. Sphere Packings, Lattices and Groups. Springer.

Dousse, O., Tavoularis, C., And Thiran, P. 2006. Delay of intrusion detection in wireless sensor networks. Proceedings of the Seventh ACM International Symposium on Mobile Ad Hoc Networking and Mobile Computing, 155-165.

FelLeR, W. 1971. An introduction to probability theory and its applications. Wiley Series in Probability and Mathematical Statistics, New York: Wiley, 1971, 3rd ed..

Flanders, H. 1989. Differential Forms With Applications to the Physical Sciences. Dover Publications.

Gui, C. And Mohapatra, P. 2004. Power conservation and quality of surveillance in target tracking sensor networks. Proceedings of the 10th Annual International Conference on Mobile Computing and Networking, 129-143.

ITô, K. 1984. Introduction to Probability Theory. Cambridge University Press.

Koushanfar, F., Slijepcevic, S., Potkonjak, M., and Sangiovanni-Vincentelli, A. 2002. Error-tolerant multimodal sensor fusion. IEEE CAS Workshop on Wireless Communication and Networking.

Kumar, S., LaI, T., And Arora, A. 2005. Barrier coverage with wireless sensors. Proceedings of the 11th Annual International Conference on Mobile Computing and Networking, 284-298.

Lazos, L. And Poovendran, R. 2006. Stochastic Coverage in Heterogeneous Sensor Networks. ACM Transactions on Sensor Networks, 325-358.

Lazos, L., Poovendran, R., And Ritcey, J. 2007a. On the Deployment of Heterogeneous Sensor Networks for Detection of Mobile Targets. Proceedings of the 5th International Symposium on Modeling and Optimization in Mobile, Ad Hoc and Wireless Networks (WiOpt'07).

Lazos, L., Poovendran, R., And Ritcey, J. 2007b. Probabilistic Detection of Mobile Targets in Heterogeneous Sensor Networks. Proceedings of the 6th International Conference on Information Processing in Sensor Networks, 519-528.

Meguerdichian, S., Koushanfar, F., Potkonjak, M., and Srivastava, M. 2001. Coverage problems in wireless ad-hoc sensor networks. Proceedings of the Twentieth Annual Joint Conference of the IEEE Computer and Communications Societies (INFOCOM 2001) 3.

Meguerdichian, S., Koushanfar, F., Qu, G., and Potkonjak, M. 2001. Exposure in wireless Ad-Hoc sensor networks. Proceedings of the 7th Annual International Conference on Mobile Computing and Networking, 139-150.

Reuleaux, F. 1963. The Kinematics of Machinery. New York: Dover.

Santaló, L. 2004. Integral Geometry and Geometric Probability. Cambridge University Press.

Solomon, H. 1978. Geometric probability. Society for Industrial and Applied Mathematics Philadelphia.

Sylvester, J. 1890. On a funicular solution of Buffon's "problem of the needle" in its most general form. Acta Mathematica 14, 1, 185-205.

YANG, H. AND SikdAR, B. 2003. A protocol for tracking mobile targets using sensor networks. Sensor Network Protocols and Applications, 2003. Proceedings of the First IEEE. 2003 IEEE International Workshop on, 71-81. 